\title{
Relating binaural pitch perception to the individual listener's auditory profile
}

\author{
Santurette, Sébastien; Dau, Torsten
}

Published in:

Acoustical Society of America. Journal

Link to article, DOI:

$10.1121 / 1.3689554$

Publication date:

2012

Link back to DTU Orbit

Citation (APA):

Santurette, S., \& Dau, T. (2012). Relating binaural pitch perception to the individual listener's auditory profile. Acoustical Society of America. Journal, 131(4, Part 1), 2968-2986. https://doi.org/10.1121/1.3689554

\section{General rights}

Copyright and moral rights for the publications made accessible in the public portal are retained by the authors and/or other copyright owners and it is a condition of accessing publications that users recognise and abide by the legal requirements associated with these rights.

- Users may download and print one copy of any publication from the public portal for the purpose of private study or research.

- You may not further distribute the material or use it for any profit-making activity or commercial gain

- You may freely distribute the URL identifying the publication in the public portal

If you believe that this document breaches copyright please contact us providing details, and we will remove access to the work immediately and investigate your claim 


\title{
Relating binaural pitch perception to the individual listener's auditory profile
}

\author{
Sébastien Santurette ${ }^{\mathrm{a})}$ and Torsten Dau \\ Centre for Applied Hearing Research, Department of Electrical Engineering, Technical University of Denmark \\ DTU Bygning 352, Ørsteds Plads, 2800 Kgs. Lyngby, Denmark
}

(Received 5 January 2011; revised 3 February 2012; accepted 6 February 2012)

\begin{abstract}
The ability of eight normal-hearing listeners and fourteen listeners with sensorineural hearing loss to detect and identify pitch contours was measured for binaural-pitch stimuli and salience-matched monaurally detectable pitches. In an effort to determine whether impaired binaural pitch perception was linked to a specific deficit, the auditory profiles of the individual listeners were characterized using measures of loudness perception, cognitive ability, binaural processing, temporal fine structure processing, and frequency selectivity, in addition to common audiometric measures. Two of the listeners were found not to perceive binaural pitch at all, despite a clear detection of monaural pitch. While both binaural and monaural pitches were detectable by all other listeners, identification scores were significantly lower for binaural than for monaural pitch. A total absence of binaural pitch sensation coexisted with a loss of a binaural signal-detection advantage in noise, without implying reduced cognitive function. Auditory filter bandwidths did not correlate with the difference in pitch identification scores between binaural and monaural pitches. However, subjects with impaired binaural pitch perception showed deficits in temporal fine structure processing. Whether the observed deficits stemmed from peripheral or central mechanisms could not be resolved here, but the present findings may be useful for hearing loss characterization.
\end{abstract}

(c) 2012 Acoustical Society of America. [http://dx.doi.org/10.1121/1.3689554]

PACS number(s): 43.66.Sr, 43.66.Pn, 43.66.Rq, 43.66.Yw [LD]

Pages: $2968-2986$

\section{INTRODUCTION}

Binaural pitch, also often termed dichotic pitch, is an auditory pitch sensation which can arise when two noise stimuli are presented simultaneously to the left and right ear, with an interaural phase shift over a specific frequency band (Cramer and Huggins, 1958). Such stimuli differ from most pitch-evoking stimuli in the sense that they require binaural interaction before any information relevant for pitch extraction can be available to the auditory system. The fact that broadband white noise, which has a flat power spectrum and does not evoke a pitch when presented monaurally, can be used to create a binaural pitch sensation, implies that such a pitch is formed centrally, using fine temporal disparities between the left and right peripheral channels. Therefore, a number of suggested models make use of interaural time differences to account for the existence of binaural pitch. These include equalization-cancellation models (e.g., Durlach, 1960; Culling et al., 1998), in which binaural cancellation occurs after the amplitude and phase of the left and right peripheral signals have been equalized, and central-spectrum models (e.g., Raatgever and Bilsen, 1986), in which a central activity pattern is computed along the two dimensions of frequency and internal interaural time delay.

Despite its relatively small salience, binaural pitch was found to be immediately perceiveable and allow melody recognition in normal-hearing $(\mathrm{NH})$ listeners (Akeroyd et al., 2001). In hearing-impaired (HI) listeners, binaural-pitch perception was found to be either as immediate as in NH lis-

\footnotetext{
a) Author to whom correspondence should be addressed. Electronic mail: ses@elektro.dtu.dk
}

teners or totally absent (Santurette and Dau, 2007). Interestingly, the two subjects who could not perceive binaural pitch at all in the latter study were the ones for whom deficits were likely to be present in central areas of the auditory system. Therefore, it was suggested that binaural pitch stimuli could be relevant to use in order to help characterize hearing loss in individual patients, because such stimuli are easy to generate and to include in a fast pitch detection test. However, a clear link between impaired binaural pitch perception and a specific auditory deficit or hearing loss type needs to be established before a binaural pitch test may be considered useful.

In addition to reduced sensitivity, sensorineural hearing loss is often accompanied by the alteration of several important properties of the normal auditory system. First, most cases of cochlear hearing loss involve damage in outer hair cells (OHCs), whose active mechanism is crucial for a sharp frequency selectivity (Ruggero and Rich, 1991). Asymmetries in OHC damage may also lead to differences in auditory-filter outputs in the two ears. This could affect binaural pitch perception by reducing the interaural correlation in internal noise representations, thereby making binaural unmasking processes less efficient (Staffel et al., 1990). Second, damage to inner hair cells (IHCs) and auditory-nerve fibers may affect the acuity of temporal fine structure (TFS) coding in HI listeners. Abnormalities in, e.g., the propagation time of the basilar-membrane traveling wave and neural phase-locking mechanisms, may contribute to impaired TFS processing (Moore, 2007). Such deficits, as well as ear asymmetries in TFS processing, may lead to a degraded representation of interaural phase differences (IPDs), and thus affect binaural pitch perception. Additionally, deficits in more central mechanisms involved in the integration and processing 
of binaural information, at or above the level of the medial superior olive, may have deleterious effects on the ability to perceive binaural pitch. Ultimately, accurate binaural pitch perception should also rely on well-functioning central pitch extraction mechanisms.

In a first investigation of the effects of hearing loss on binaural pitch perception, Santurette and Dau (2007) showed that the detection and melody recognition scores of HI listeners with binaural pitch stimuli were not correlated with lowfrequency pure-tone hearing thresholds. Their findings also demonstrated that impaired frequency selectivity affected pitch salience, but that estimates of auditory-filter bandwidths were not correlated with melody recognition abilities with binaural pitch stimuli. Nitschmann et al. (2010) also showed that, even though HI listeners who were unable to perceive binaural pitch had elevated auditory filter bandwidths, other HI listeners with similar bandwidths showed an immediate binaural pitch sensation. Therefore, impaired frequency selectivity alone cannot explain the inability of some listeners to perceive binaural pitch.

As no spectral cues are present in the physical stimulus, binaural pitch perception can be expected to rely heavily on accurate TFS processing and intact binaural integration mechanisms. However, so far it has not been investigated whether the inability of some HI listeners to perceive binaural pitch reflects a deficit in binaural processing per se, which would be located at the level of the brainstem or at higher auditory stages, or if reduced temporal acuity in the periphery is sufficient to account for impaired binaural pitch perception. The results of Strelcyk and Dau (2009), who showed a significant correlation between binaural and monaural measures of TFS processing in HI listeners, suggested that an impaired monaural representation of TFS might account for the binaural deficits. Moreover, TFS processing outcomes were not correlated to measures of frequency selectivity in their study. In addition, Nitschmann et al. (2010) found that the ratio of binaural to monaural estimates of frequency selectivity did not differ between NH and HI listeners, suggesting that the deficits apparent in binaural measures reflect a basic peripheral auditory impairment, rather than a specific binaural impairment.

The present study related the ability of HI listeners to process binaural pitch stimuli to their performance in an extensive set of specific measures of basic auditory and cognitive functions, referred to as the "auditory profile" in the following. A first aim was to compare the effects of hearing impairment on the perception of binaurally $v s$. monaurally elicited noise pitches, and to relate such effects to deficits in basic auditory functions. A second aim was to investigate the relationship between the different auditory-profile measures as such, as an analysis of correlations between the outcomes of the different tests might help better understand the auditory processes involved in sensorineural hearing loss, and at which level of the auditory system they may take place. Here, the approach was to perform an extensive set of tests on a heterogeneous group of HI listeners, with the postulate that pointing out individual differences might be as informative as focusing on group averages. While the tested subject group may reflect the diversity of impairments found in the HI population, this approach does not provide a direct assessment of the diagnostic value of a binaural pitch test in a clinical setting. Instead, it was attempted here to determine whether binaural pitch is an informative tool that may complement or replace other existing measures when attempting to characterize hearing loss.

Binaural pitch perception was evaluated in two experiments (Sec. III). The first short test involved detection of a musical scale played with binaural pitch stimuli. The second, more extensive, test evaluated detection and identification of pitch contours played either with binaural pitch stimuli, or with pitch-evoking noise stimuli for which the pitch was detectable monaurally. In addition to being a more accurate measure of binaural-pitch detection, the comparison of binaural and monaural identification scores in this second test allowed to make the distinction between a binaural deficit and a more general difficulty in extracting tonal objects from noise (Chait et al., 2007).

The test battery used to characterize the listeners' auditory profiles was designed to evaluate basic functions, which may be affected by hearing loss. The battery included the common audiometric measures (Sec. IV): pure-tone air-conduction and bone-conduction thresholds, tympanograms and stapedius reflex responses to test for the presence of middle ear dysfunction and potential auditory or facial nerve disorders, and click-evoked otoacoustic emissions to further assess cochlear function. Loudness perception estimates (Sec. V) were also included, whereby a set of loudness curves was obtained using a categorical loudness scaling procedure. This was used to determine adequate presentation levels in other tests, but also in order to evaluate recruitment. The loudness growth measures were supplemented with a standard intensity-increment detection test, to better assess the presence of cochlear and retrocochlear disorders. The cognitive abilities of the listeners were evaluated (Sec. VI) using a lexical decision task and a reading span test, in order to estimate the possible influence of non-auditory deficits on the different tasks. The ability of the listeners to take advantage of binaural processing when extracting signals from noise was then evaluated, using two measures of binaural unmasking (Sec. VII): Binaural masked detection thresholds of sinusoidal tones in band-limited noise were compared for homophasic and antiphasic tones, and the spatial release from masking was evaluated in monaural and binaural conditions for speech reception thresholds (SRTs) in speechshaped noise. The latter measure was chosen because it did not only allow for evaluation of binaural processing, but also of speech intelligibility and spatial release from masking, within a single experiment. The ability of the listeners to use TFS cues in quiet was tested both binaurally and monaurally (Sec. VIII): Binaural TFS processing abilities were evaluated via detection of an IPD in a sinusoidal tone as a function of frequency, and frequency-modulation (FM) detection at a low FM-rate was used as a measure of monaural TFS processing. Finally, monaural frequency selectivity was investigated and the left and right auditory filter shapes were estimated from the results of a notched-noise experiment (Sec. IX). A detailed description of all tests is provided in the Appendix of this paper. 


\section{METHODS}

\section{A. Subjects}

Eight normal-hearing (NH) subjects (median age: 25 years) and 14 hearing-impaired (HI) subjects with sensorineural hearing-loss (median age: 64.5 years) participated in the study, which was approved by the Science-Ethics Committee for the Capital Region of Denmark (reference H-KA04149-g). The HI listeners were recruited via a newspaper advertisement. All NH subjects had audiometric thresholds equal to or below $20 \mathrm{~dB}$ hearing level (HL) at all tested frequencies. Audiograms of HI subjects are given in Fig. 1. Except for subjects 11 and 14, all HI subjects had symmetric or nearly symmetric audiograms and mean low-frequency hearing thresholds below $45 \mathrm{~dB}$ HL. Throughout the paper, "low-frequency hearing thresholds" refer to the average hearing thresholds between 125 and $2000 \mathrm{~Hz}$ over both ears. As no individual clinical diagnoses were available, the subjects were asked to report the origin of their hearing loss verbally. These self-reported origins of hearing loss, mean lowfrequency hearing thresholds, as well as the gender and age of each subject are listed in Table I.

\section{B. Experimental set-up}

All measurements were carried out via a PC in a doublewalled sound-attenuating listening booth. Unless otherwise specified, all test procedures were implemented in MATLAB, the stimuli were presented through Sennheiser HDA200 audiometric headphones connected to an RME DIGI 96/8 24-bit D/A converter, and a $48-\mathrm{kHz}$ sampling frequency was used. Calibration was performed using a B\&K 2636 sound level meter, a B\&K 4153 artificial ear, and a B\&K 4230 artificial-ear calibrator. Additionally, 128-tap linear-phase FIR equalization filters were applied to all broadband stimuli, in order to flatten the headphone frequency response. Testing was divided in experimental sessions of maximum $2 \mathrm{~h}$, with no more than one session per day per listener. The total testing time was around 9-10 hours per listener. The audiometric measures were performed first, followed by the loudness perception tests and the binaural pitch experiments.
The remaining tests were then conducted in the same order as presented below.

\section{BINAURAL PITCH EXPERIMENTS}

Two pitch-evoking noise stimuli were generated: a binaural pitch (BP) stimulus, and a similar-sounding stimulus evoking a monaurally detectable pitch (MP). The BP stimulus contained a Huggins' pitch, as this configuration has been reported to evoke the most salient pitch among different types of binaural pitches (Akeroyd et al., 2001; Santurette and Dau, 2007). The pitch in the MP stimulus was created by raising the amplitude of the noise in both ears over a narrow frequency range. The total noise bandwidth was $4 \mathrm{kHz}$ for all stimuli and the transition band of the BP stimulus, over which the interaural phase difference varied linearly from 0 to $2 \pi$, had a bandwidth equal to $16 \%$ of the band's center frequency.

\section{A. Scale test}

\section{Method}

In this short test, the listeners were presented with a sequence of 10 musical notes forming a major scale, ranging from $\mathrm{C}_{5}(523.25 \mathrm{~Hz})$ to $\mathrm{C}_{6}(1046.50 \mathrm{~Hz})$, played with the $\mathrm{BP}$ stimulus. The procedure and note frequencies were identical to those described in Santurette and Dau (2007), except that 1 -ms ramps were used between intervals, such that the 10-s noise stimulus was perceived as continuous. After one presentation of the stimulus, subjects were asked to verbally report whether something else than noise could be perceived. The test was first performed at an overall level equal to the binaurally-measured most comfortable level (MCL) at $500 \mathrm{~Hz}$ (see Sec. V and Table I). If the test was negative, i.e., only noise was perceived, it was repeated at MCL $+10 \mathrm{~dB}$, and if still negative, at MCL $+20 \mathrm{~dB}$.

\section{Results}

The individual results are summarized in Table I. "Y" indicates that binaural pitch was immediately perceived at MCL, i.e., that the listener reported hearing a pitch within

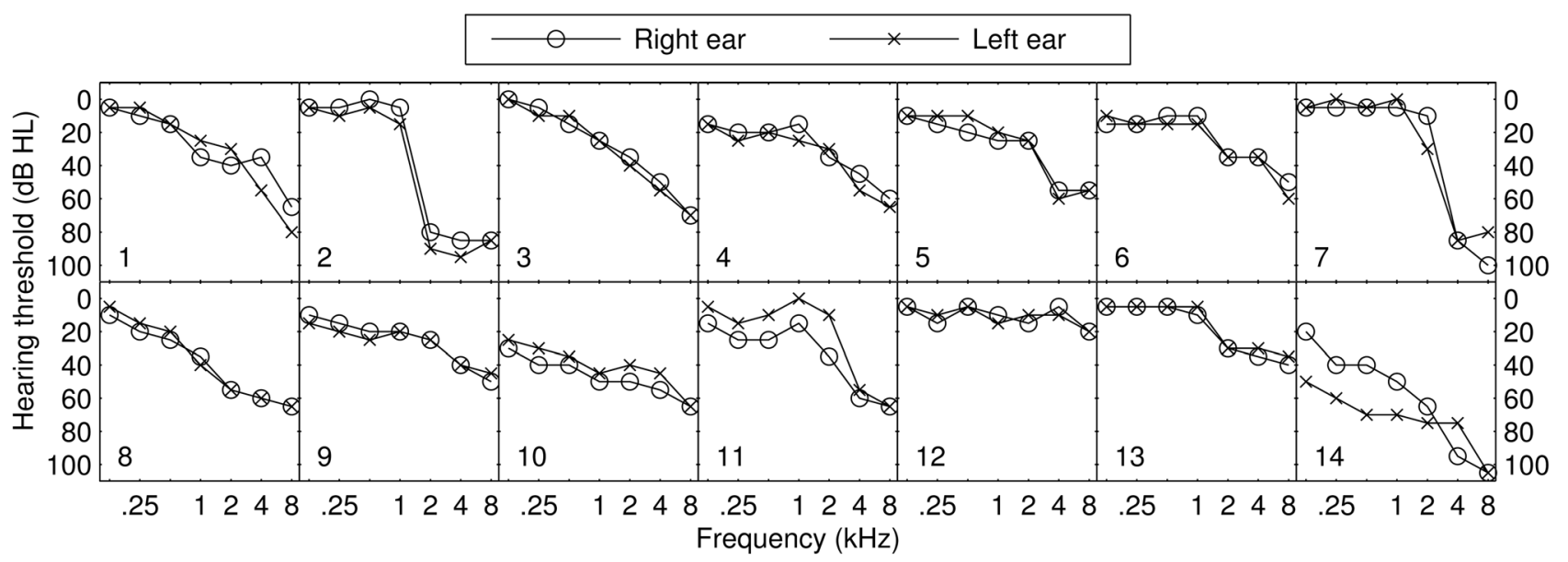

FIG. 1. Air-conduction pure-tone hearing thresholds of the 14 hearing-impaired subjects. Subject number is indicated at the bottom left of each audiogram. 
TABLE I. From left to right. Subject ID (letters for NH and numbers for HI subjects); gender (f for female, m for male); age on first day of study; musical experience $(\mathrm{M})$ : + denotes a musically trained listener; self-reported origin of hearing loss; mean right-ear (R) and left-ear (L) hearing threshold between 125 and $2000 \mathrm{~Hz}$ (LF-HL), in dB HL; right (R) and left (L) tympanogram type; ipsilateral (I) and contralateral (C) acoustic reflex measured in the right (R) and left (L) ear: + denotes a clear response, \pm a weak response, and - an absent response; overall SNR of CEOAE response in the right (R) and left (L) ear: + denotes a clear response, \pm a weak response, and - an absent response; results of the binaural pitch scale test: Y indicates that the scale was immediately perceived at MCL, NY at MCL +10, NNY at MCL+20, and NNN indicates that only noise was perceived at the three levels; binaural most comfortable level (BMCL) at 0.5 and $1 \mathrm{kHz}$, in $\mathrm{dB}$ SPL; binaural (B), right-ear (R), and left-ear (L) $L_{10}$ levels at 0.5 and $1 \mathrm{kHz}$, in dB SPL; relative asymmetry of FM detection thresholds (FM-As.) at 0.5 and $1 \mathrm{kHz}$ (in \%); relative asymmetry of the ERB of auditory filter estimates at $0.5 \mathrm{kHz}$ (in \%).

\begin{tabular}{|c|c|c|c|c|c|c|c|c|c|c|c|c|c|c|c|c|c|c|c|c|c|c|c|c|c|c|}
\hline \multirow[b]{2}{*}{ \# } & \multirow[b]{2}{*}{ Sex } & \multirow[b]{2}{*}{ Age } & \multirow[b]{2}{*}{ M } & \multirow{2}{*}{$\begin{array}{l}\text { Self-reported } \\
\text { origin }\end{array}$} & \multicolumn{2}{|c|}{ LF-HL } & \multicolumn{2}{|c|}{ Tymp. } & \multicolumn{2}{|c|}{ I-reflex } & \multicolumn{2}{|c|}{ C-reflex } & \multicolumn{2}{|c|}{ OAE-SNR } & \multirow[b]{2}{*}{ Scale } & \multicolumn{2}{|c|}{ BMCL } & \multicolumn{2}{|c|}{$\mathrm{B}-L_{10}$} & \multicolumn{2}{|c|}{$\mathrm{R}-L_{10}$} & \multicolumn{2}{|c|}{$\mathrm{L}-L_{10}$} & \multicolumn{2}{|c|}{ FM-As. } & \multirow{2}{*}{$\begin{array}{c}\text { ERB } \\
\text { As. }\end{array}$} \\
\hline & & & & & $\mathrm{R}$ & $\mathrm{L}$ & $\mathrm{R}$ & $\mathrm{L}$ & $\mathrm{R}$ & $\mathrm{L}$ & $\mathrm{R}$ & $\mathrm{L}$ & $\mathrm{R}$ & $\mathrm{L}$ & & $0.5 \mathrm{k}$ & $1 \mathrm{k}$ & $0.5 \mathrm{k}$ & $1 \mathrm{k}$ & $0.5 \mathrm{k}$ & $1 \mathrm{k}$ & $0.5 \mathrm{k}$ & $1 \mathrm{k}$ & $0.5 \mathrm{k}$ & $1 \mathrm{k}$ & \\
\hline A & $\mathrm{m}$ & 24.6 & + & none & 0 & 2 & A & A & + & + & + & + & $4.5+$ & $3.1+$ & $\mathrm{Y}$ & 77 & 74 & 47 & 45 & 44 & 39 & 45 & 40 & 8 & 2 & 18 \\
\hline B & $\mathrm{m}$ & 25.6 & + & none & 0 & -2 & A & A & + & + & + & + & $3.9+$ & $5.8+$ & $\mathrm{Y}$ & 66 & 65 & 31 & 29 & 41 & 29 & 35 & 25 & 1 & 13 & 15 \\
\hline $\mathrm{C}$ & $\mathrm{m}$ & 21.1 & + & none & -4 & -6 & A & A & + & + & + & + & $3.3+$ & $2.0+$ & $\mathrm{Y}$ & 58 & 57 & 30 & 30 & 35 & 30 & 33 & 31 & 15 & 17 & 17 \\
\hline $\mathrm{D}$ & $\mathrm{f}$ & 21.7 & - & none & -1 & 0 & A & A & + & + & + & + & $3.1+$ & $1.7+$ & $\mathrm{Y}$ & 72 & 85 & 46 & 47 & 53 & 49 & 52 & 48 & 10 & 24 & 1 \\
\hline $\mathrm{E}$ & $\mathrm{f}$ & 47.3 & + & none & 2 & 2 & A & A & + & + & + & + & $2.1+$ & $2.9+$ & $\mathrm{Y}$ & 77 & 63 & 47 & 37 & 46 & 45 & 51 & 45 & 19 & 17 & 15 \\
\hline $\mathrm{F}$ & $\mathrm{m}$ & 26.7 & + & none & 2 & 1 & A & A & + & + & + & + & $2.8+$ & $2.2+$ & $\mathrm{Y}$ & 64 & 54 & 39 & 28 & 39 & 33 & 41 & 31 & 12 & 20 & 13 \\
\hline G & $\mathrm{f}$ & 24.2 & + & none & 0 & -1 & A & A & + & + & + & + & $2.9+$ & $2.6+$ & $\mathrm{Y}$ & 70 & 62 & 45 & 31 & & & & & & & \\
\hline $\mathrm{H}$ & $\mathrm{m}$ & 25.3 & - & none & -1 & -2 & A & A & + & + & + & + & $8.6+$ & $8.7+$ & $\mathrm{Y}$ & 66 & 63 & 33 & 31 & & & & & & & \\
\hline 1 & $\mathrm{f}$ & 67.6 & + & unknown & 24 & 20 & A & $\mathrm{A}$ & \pm & + & - & + & $1.9-$ & $1.2-$ & $\mathrm{Y}$ & 71 & 76 & 48 & 55 & 32 & 56 & 56 & 31 & 9 & 5 & 2 \\
\hline 2 & $\mathrm{f}$ & 46.5 & - & innate & 18 & 25 & A & $\mathrm{A}$ & + & - & + & - & $8.4+$ & $8.4+$ & $\mathrm{Y}$ & 76 & 61 & 46 & 34 & 41 & 40 & 46 & 47 & 61 & 14 & 1 \\
\hline 3 & $\mathrm{~m}$ & 66.3 & - & unknown & 19 & 19 & A & A & + & + & + & + & $3.1+$ & $1.4-$ & NY & 66 & 67 & 47 & 48 & 50 & 49 & 48 & 49 & 9 & 12 & 11 \\
\hline 4 & f & 64.8 & - & unknown & 21 & 24 & A & $\mathrm{A}$ & + & + & + & + & $2.5+$ & $1.8-$ & NY & 73 & 70 & 53 & 47 & 52 & 54 & 59 & 49 & 5 & 8 & 9 \\
\hline 5 & $\mathrm{~m}$ & 68.7 & - & noise exposure & 20 & 17 & A & A & + & + & + & + & $1.4+$ & $1.0-$ & NY & 72 & 75 & 50 & 54 & 54 & 52 & 51 & 59 & 11 & 2 & 6 \\
\hline 6 & f & 66.3 & - & unknown & 17 & 19 & A & A & + & + & + & + & $1.9+$ & $2.7+$ & $\mathrm{Y}$ & 76 & 70 & 51 & 46 & 51 & 52 & 54 & 53 & 12 & 16 & 4 \\
\hline 7 & $\mathrm{~m}$ & 59.4 & - & noise exposure & 6 & 8 & A & As & \pm & \pm & \pm & \pm & $1.9 \pm$ & $2.0-$ & $\mathrm{Y}$ & 76 & 76 & 53 & 47 & 56 & 46 & 57 & 45 & 14 & 1 & 2 \\
\hline 8 & $\mathrm{~m}$ & 65.5 & - & unknown & 29 & 27 & A & A & + & + & + & + & $0.6-$ & $1.2-$ & NNN & 72 & 71 & 50 & 57 & 46 & 50 & 42 & 51 & 29 & 10 & 9 \\
\hline 9 & $\mathrm{~m}$ & 55.1 & - & high-voltage shock & 19 & 21 & A & $\mathrm{C}$ & + & + & + & + & $3.5+$ & $1.2-$ & NY & 71 & 75 & 51 & 50 & 52 & 48 & 49 & 49 & 11 & 0 & 22 \\
\hline 10 & $\mathrm{f}$ & 63.7 & + & hereditary & 43 & 36 & A & A & + & \pm & + & \pm & $1.3-$ & $1.3-$ & NNN & 84 & 82 & 64 & 65 & 68 & 72 & 64 & 65 & 24 & 10 & 37 \\
\hline 11 & $\mathrm{~m}$ & 63.3 & + & neural disorder & $23^{\mathrm{a}}$ & $8^{\mathrm{a}}$ & A & A & + & + & + & + & $1.0-$ & $1.0-$ & NY & 74 & 69 & 51 & 40 & 66 & 60 & 59 & 52 & 19 & 6 & 16 \\
\hline 12 & $\mathrm{f}$ & 35.6 & - & brain trauma & 10 & 9 & A & A & + & + & + & + & $8.1+$ & $3.6+$ & $\mathrm{Y}$ & 71 & 67 & 47 & 43 & 37 & 39 & 39 & 39 & 12 & 8 & 14 \\
\hline 13 & $\mathrm{f}$ & 56.2 & + & brain trauma & 11 & 10 & A & $\mathrm{A}$ & + & + & + & + & $3.9+$ & $4.7+$ & $\mathrm{Y}$ & 61 & 62 & 38 & 36 & 46 & 48 & 47 & 47 & 0 & 16 & 20 \\
\hline 14 & $\mathrm{f}$ & 66.5 & - & brain trauma & $45^{\mathrm{a}}$ & $66^{\mathrm{a}}$ & A & $\mathrm{A}$ & - & \pm & - & - & $0.8-$ & $0.7-$ & NNN & 79 & 79 & 67 & 67 & 66 & 64 & 93 & 78 & 9 & 7 & 6 \\
\hline
\end{tabular}

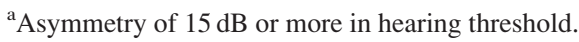

the noise after the first stimulus presentation. "NY" indicates that a pitch was first heard during the second presentation $(\mathrm{MCL}+10)$, and "NNN" that no pitch was heard in any of the three presentations, i.e., the listener reported hearing noise only, even at MCL +20 . All NH listeners and six HI listeners could hear binaural pitch immediately at MCL, while five other $\mathrm{HI}$ listeners could first perceive it at MCL +10 . The three remaining HI listeners $(8,10$, and 14) only perceived noise at all presentation levels, suggesting impaired binaural-pitch perception.

\section{B. Pitch contour detection and identification}

\section{Method}

The ability of the listeners to detect and identify pitch contours generated with BP and MP stimuli were measured and compared, using a procedure similar to that described in Santurette et al. (2010). In each trial, a sequence of five musical notes [Table II(a)], corresponding to frequencies within the range of strongest salience of Huggins' pitch (Santurette and Dau, 2007), were presented to the listeners, such that they formed one of the five possible pitch contours listed in Table II(b). The task of the subjects was to press one of six buttons on a computer screen after each stimulus presentation. Five buttons corresponded to the five possible pitch con- tours, represented by symbols, while the words "no melody" appeared on the sixth button. The listeners were instructed to press the "no melody" button if no pitch was heard at all. If any pitch was heard, they were asked to press the symbol corresponding to the perceived pitch contour. The "no melody" option was included so that both detection and contour recognition could be tested within a single experiment.

The BP and MP stimuli were generated and adjusted for equivalent pitch salience as described in Santurette et al. (2010). In that study, following a salience-adjustment experiment in NH listeners, a linear relationship ${ }^{1}$ was derived between the broadband-noise level and the level of the additional narrow-band noise in MP producing an equally-salient

TABLE II. Note frequencies and pitch contours used in the pitch contour identification experiment. (a) Note frequencies. (b) Pitch contours.

\begin{tabular}{lcccc}
\hline \multicolumn{2}{c}{ (a) Note frequencies } & & \multicolumn{2}{c}{ (b) Pitch contours } \\
\cline { 1 - 1 } Note & Frequency & & Contour & Note sequence \\
\hline $\mathrm{C}_{5}$ & $523.25 \mathrm{~Hz}$ & & Rising only & $\mathrm{C}_{5}-\mathrm{D}_{5}-\mathrm{E}_{5}-\mathrm{F}_{5}-\mathrm{G}_{5}$ \\
$\mathrm{D}_{5}$ & $587.32 \mathrm{~Hz}$ & & Falling only & $\mathrm{G}_{5}-\mathrm{F}_{5}-\mathrm{E}_{5}-\mathrm{D}_{5}-\mathrm{C}_{5}$ \\
$\mathrm{E}_{5}$ & $659.26 \mathrm{~Hz}$ & & Rising then falling & $\mathrm{C}_{5}-\mathrm{D}_{5}-\mathrm{E}_{5}-\mathrm{D}_{5}-\mathrm{C}_{5}$ \\
$\mathrm{~F}_{5}$ & $698.46 \mathrm{~Hz}$ & & Falling then rising & $\mathrm{G}_{5}-\mathrm{F}_{5}-\mathrm{E}_{5}-\mathrm{F}_{5}-\mathrm{G}_{5}$ \\
$\mathrm{G}_{5}$ & $783.99 \mathrm{~Hz}$ & & Constant & $\mathrm{E}_{5}-\mathrm{E}_{5}-\mathrm{E}_{5}-\mathrm{E}_{5}-\mathrm{E}_{5}$ \\
\hline \hline
\end{tabular}


pitch to that of the corresponding BP stimulus. The same linear relationship was used here, with the level of the broadband noise individually adjusted to the binaurally-measured MCL at $500 \mathrm{~Hz}$ (Table I). 30-ms onset and offset ramps were applied to the overall stimulus. Three different note durations were used $(300,600$, and $900 \mathrm{~ms})$. Subjects were presented 30 trials for each combination of stimulus type and note duration. Each possible pitch contour was presented an equal number of times. In addition to these 180 trials containing a pitch contour, 36 trials containing no pitch contour (diotic white noise only) were presented, one third of them corresponding to each duration. This made it possible to evaluate false alarms and to avoid the possibility of subjects never pressing the "no melody" button. The experiment was divided into two blocks and trials were presented in a random order within each block. Before these two experimental blocks, each subject was first introduced to the different pitch contours played with pure-tone stimuli. In addition, at least one short 18-trial practice block was performed with pure tones to ensure that the task was correctly understood. Subjects were not informed about the existence of different stimulus types.

\section{Results and discussion}

All NH listeners and $11 \mathrm{HI}$ listeners obtained overall detection scores above $95 \%$ for both MP and BP stimuli, indicating a clear pitch sensation, independent of the use of binaural or monaural cues. HI subject 11 was also able to perceive both pitch types clearly (MP: $86 \%$, BP: $81 \%$ ). The two remaining HI listeners could perceive MP in $97 \%$ of trials, but failed to perceive BP stimuli (subject 10: 1\%, subject 14: $0 \%$ ). Despite his negative response in the scale test, subject 8 was clearly able to detect BP as well as MP. This suggests that a negative response in the scale test is not sufficient to conclude that binaural-pitch perception is absent, which was also observed by Nitschmann et al. (2010) in one of their subjects. In summary, for HI listeners, the BP stimulus was either as easy to detect as the MP stimulus, or not perceived at all despite accurate MP detection. This rules out a general difficulty in extracting tonal objects from background noise in HI listeners unable to perceive binaural pitch, and confirms that the lack of accurate binaural information must be a crucial factor in the observed deficit. Stimulus duration was found to have no effect on the detection scores of HI listeners (MP: $p=0.349$, BP: $p=0.379$, Kruskal-Wallis test). Low false alarm rates, i.e., proportions of trials containing no pitch contour in which another button than "no melody" was pressed, were obtained in most subjects (mean $2.8 \%$, median $1.0 \%$ ). Individual false alarm rates higher than $20 \%$ were obtained in subject $\mathrm{A}$ (36\%), subject $2(36 \%)$, and subject 7 (22\%).

The ability of the listeners to correctly identify the pitch contours is represented in Fig. 2. The proportions of trials containing a pitch contour in which the pitch contour was correctly identified are plotted for the MP and BP stimuli against each other. Here and in all following figures, the relevant individual results are indicated using letters for NH subjects and numbers for HI subjects (see Table I). NH listeners

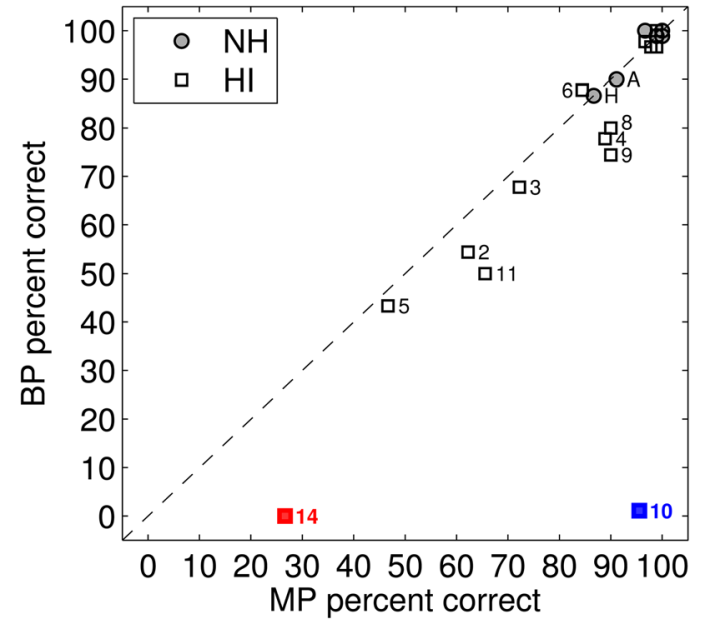

FIG. 2. (Color online) Pitch contour identification scores for the MP (horizontal axis) and BP (vertical axis) stimuli, expressed as the percentage of trials containing a pitch contour in which the pitch contour was correctly identified.

all obtained identification scores above $85 \%$ and similar results for the MP and BP stimuli. A larger variability was found in the HI group, with five listeners showing similar performance to the NH group (subjects 1, 6, 7, 12, and 13), and seven other listeners obtaining scores below $80 \%$ either for the BP stimulus only or for both stimulus types. For the latter group of subjects, BP identification was always poorer than MP identification (points below the diagonal line in Fig. 2), despite similar detection scores for MP and BP. This difference was significant $(p=0.017$, Wilcoxon paired signed-rank test, $\alpha=5 \%$ here and throughout the paper), indicating a reduced salience or musicality of BP compared to MP for these listeners. The two HI subjects who could not perceive binaural pitch performed very differently in identifying MP contours: Subject 14 showed difficulty in pitch contour identification, while subject 10 did not. No effect of stimulus duration was found on pitch contour identification in the HI group (MP: $p=0.458$, BP: $p=0.726$, Kruskal-Wallis test).

Overall pitch identification scores of the HI listeners (MP score for subjects 10 and 14, mean MP and BP score for all other subjects) were significantly correlated to pitch contour identification with pure-tone stimuli obtained in the practice blocks of the experiment $(p=0.002, \rho=0.75)$. This suggests that the low salience of MP and BP, in comparison to that of pure tones, was not the main factor responsible for reduced pitch contour identification in some HI listeners. Only for subjects 5 and 14 were pure-tone scores much higher than MP and BP scores, which suggests a detrimental effect of using noise-based stimuli for these two specific subjects. The pitch contour identification abilities of the listeners were overall not found to rely on their musical experience (column "M" in Table I).

In addition to binaural pitch detectability, two additional outcomes of this test were used to perform a correlation analysis with the different measures of the auditory profile described further below: average pitch contour identification scores, as well as the difference in performance between MP and BP identification scores as an estimate of the binaural disadvantage for pitch contour identification. In the following, 
"MP + BP scores" thus refer to the average pitch identification scores of HI listeners with MP and BP stimuli (except for subjects 10 and 14, for whom it refers to performance with the MP simulus only). For listeners who could perceive binaural pitch, the "MP - BP score" refers to the difference between the MP and BP scores divided by the MP + BP score. All correlation coefficients mentioned in this paper correspond to Spearman's $\rho$ and, unless specified otherwise, are calculated for the HI group. No correction was applied to the reported $p$-values, ${ }^{2}$ which were calculated from the exact permutation distributions.

In addition to the correlations of $\mathrm{MP}+\mathrm{BP}$ and $\mathrm{MP}-\mathrm{BP}$ scores with the different outcomes, the performance of the two subjects who could not perceive binaural pitch at all (10 and 14) in the different auditory profile measures will be in focus in Secs. IV-IX. The results of subjects 9 and 11 may also be of particular interest, because these two listeners obtained the highest MP - BP scores in the HI group (data points furthest away from the diagonal line in Fig. 2).

The detailed methods of the auditory profile tests presented in the following can be found in the Appendix of this paper.

\section{AUDIOMETRIC MEASURES}

\section{A. Pure-tone audiometry}

Pure-tone air-conduction audiograms of all HI subjects are given in Fig. 1. None of the listeners showed an air-bone gap, indicating no sign of conductive dysfunction. All hearing losses were thus of the sensorineural type. Subject 12 showed no hearing-threshold elevation at any audiometric frequency, and is thus to be classified as suffering from an obscure dysfunction (e.g., Saunders and Haggard, 1989; King and Stephens, 1992; Strelcyk and Dau, 2009).

It is worth noting that the two listeners who could not perceive BP (10 and 14) were also the ones with the highest average low-frequency hearing thresholds (LF-HL in Table I). This raises the question of the influence of sensation level on BP detection scores. However, the use of the MCL as a testing level should have ensured sufficient audibility in the binaural pitch tests, and neither subject 10 nor 14 benefited from a raised sensation level in the scale test. Moreover, in the study of Nitschmann et al. (2010), some listeners with moderate low-frequency hearing loss, similar to that of subject 10 in the present study, were able to perceive binaural pitch. Therefore, it is unlikely that audibility alone was responsible for the absence of binaural pitch percept found in subjects 10 and 14 . Neither MP + BP scores nor MP - BP scores were correlated with mean hearing thresholds at 0.5 and $1 \mathrm{kHz}(\mathrm{MP}+\mathrm{BP}$ : $p=0.234$, MP $-\mathrm{BP}: p=0.499)$.

\section{B. Tympanometry and acoustic reflex}

Tympanograms as well as ipsilateral and contralateral stapedius reflex curves were obtained in both ears for each subject. The obtained tympanogram types, as described in Gelfand (2001a) using the classification of Jerger (1970), are given in Table I. All subjects obtained type A tympanograms in both ears, except subject 7 who showed a shallow admittance peak in the left ear (type As), and subject 9 who showed a negative-pressure peak in the left ear (type C). Together with the absence of an air-bone gap, this confirms the sensorineural nature of the hearing loss in all listeners. Although type $\mathrm{C}$ tympanograms are usually associated with Eustachian tube dysfunction (Feldman, 1977; Gelfand, 2001a), the symmetry in the audiogram of subject 9 suggests that this did not affect his hearing ability.

The nature of the individual stapedius reflex responses are given in Table I for ipsilateral (I-reflex) and contralateral (C-reflex) stimulation. A "+" sign indicates the presence of a clear response at a presentation level of $100 \mathrm{~dB}$ sound pressure level (SPL) or below for at least one of the three test frequencies $(0.5,1$, and $2 \mathrm{kHz})$. A “-” sign indicates that no response was obtained at $100 \mathrm{~dB}$ SPL for any test frequency. A “土” sign indicates a weak or undetermined response. Most HI listeners obtained patterns of responses which did neither suggest a conductive dysfunction nor a deficit along the neural sensory pathways. Only subject 2 obtained a clear pattern usually associated with VIIIth nerve disorder on the left side (Gelfand, 2001a). The absence of a clear response for subject 14 may reflect her elevated hearing threshold compared to other subjects, as auditory reflex thresholds above $100 \mathrm{~dB}$ SPL are not uncommon in cases of moderate to moderately severe sensorineural hearing loss (Gelfand, 2001a).

\section{Otoacoustic emissions}

Click-evoked otoacoustic emissions (CEOAEs) were measured in both ears for each subject. The frequency spectrum of the CEOAE response and the noise-floor spectrum were plotted and compared for each measured ear. An artifact-rejection template was applied to remove around $10 \%$ of the noisiest epochs, in order to increase the signalto-noise ratio (SNR), before the click responses were averaged. The obtained overall SNRs are given in Table I, together with a visual assessment of the strength of the response at low frequencies $(500$ to $1500 \mathrm{~Hz})$. A “+" sign indicates a clearly visible response, a " \pm " sign a weak or undetermined response, and a "_" sign a response which was not distinguishable from the noise floor. While OAEs were present in all NH ears, only HI listeners 2, 6, 12, and 13 showed a clearly visible response in both ears, suggesting the presence of normal $\mathrm{OHC}$ function over at least a large part of the low-frequency range. This is consistent with the hearing thresholds of these subjects lying within the $\mathrm{NH}$ range up to at least $1 \mathrm{kHz}$, as CEOAEs are present in about $98 \%$ of $\mathrm{NH}$ ears and in more than $95 \%$ of HI ears with sensorineural hearing loss where the hearing threshold lies below $18 \mathrm{~dB}$ HL (Probst et al., 1991). The two listeners with absent binaural pitch perception showed no OAE response, which was expected as their hearing thresholds exceeded $35 \mathrm{~dB}$ HL (Probst et al., 1991). The OAE SNRs in the HI group were significantly correlated with the low-frequency hearing thresholds in the corresponding ears $(p=0.008$, $\rho=-0.49$ ), and so were the mean SNRs with the age of the HI listeners $(p=0.006, \rho=-0.70)$. 


\section{LOUDNESS PERCEPTION}

\section{A. Loudness scaling}

An adaptive categorical loudness scaling procedure (e.g., Brand and Hohmann, 2002; ISO 16832, 2006) was used to determine the loudness function and most-comfortable level (MCL). Measurements were performed binaurally at 500, 1000 , and $3000 \mathrm{~Hz}$, and monaurally at 500 and $1000 \mathrm{~Hz}$. The MCL in a given condition was defined as the level corresponding to 20 loudness categorical units (cu). The loudness scaling experiment was mainly used as a tool to determine adequate presentation levels in individual HI listeners. These are provided in Table I for test frequencies of 0.5 and $1 \mathrm{kHz}$, in the form of the binaurally measured MCL (BMCL), and in the form of the levels corresponding to 10 loudness $\mathrm{cu}\left(L_{10}\right)$ for binaural as well as monaural left and right presentations.

In addition to ensuring sufficient audibility of the stimuli in the different experiments, loudness curves may also be used to evaluate the degree of loudness recruitment in each listener. This may help shed light on the location of the individual hearing losses, because loudness recruitment has been associated with a cochlear site of lesion in sensorineural hearing loss (Dix et al., 1948). However, much caution is needed as recruitment is also found in cases of neural auditory disorders (Priede and Coles, 1974) and has been proven to be a weak predictor as a site-of-lesion test (Hood, 1969). The loudness growth at threshold was estimated as the slope, in $\mathrm{cu} / \mathrm{dB}$, of the lower section of the fitted loudness curve for each measurement. The obtained values for the binaural measurements at $0.5,1$, and $3 \mathrm{kHz}$ are plotted in the left panel of Fig. 3. For readability reasons, "rs" ("remaining subjects") is used to indicate the mean result of HI listeners not mentioned by their number, as these listeners obtained very similar values. As the left and right loudness-growth values at 0.5 and $1 \mathrm{kHz}$ were found to show a high degree of symmetry and follow the same trend as the binaurally measured values, only the binaural loudness growth is shown in Fig. 3 .
At 0.5 and $1 \mathrm{kHz}$, most $\mathrm{HI}$ listeners had normal or nearnormal loudness growth, which may be accounted for by the fact that most of them only had elevated hearing thresholds at high frequencies. Reflecting this, the binaural loudness growth values in the HI group were significantly correlated with the average hearing thresholds over both ears for all three test frequencies $(500 \mathrm{~Hz}: p=0.007, \rho=0.68 ; 1 \mathrm{kHz}$ : $p<0.001, \rho=0.97 ; 3 \mathrm{kHz}: p=0.038, \rho=0.56)$. This is consistent with the findings of Al-Salim et al. (2009), who reported loudness growth values that were correlated with hearing thresholds. While these loudness growth estimates may thereby not reveal much about the site of lesion for most listeners, the difference in loudness growth for subjects 10 and 14 is worth mentioning. While subject 14 showed clear recruitment, loudness growth was substantially lower for subject 10 , who obtained a value similar to that of subject 12 at $3 \mathrm{kHz}$, despite a difference of about $40 \mathrm{~dB}$ in hearing threshold between these two listeners. According to this, it is likely that a cochlear impairment is present in subject 14 , but not in subject 10 .

\section{B. Short increment sensitivity index}

A Short Increment Sensitivity Index (SISI) test (Jerger et al., 1959) was performed in the left and right ear at 500, 1000 , and $3000 \mathrm{~Hz}$. The results may be useful to further assess the presence of loudness recruitment. The right panel of Fig. 3 shows the SISI scores obtained by the individual listeners in each ear for the three test frequencies. The listeners indicated by "rs" all obtained the same score. The test results are classified as positive $(\geq 80 \%)$, questionable (25-75\%), or negative ( $\leq 20 \%)$, following the stricter of several possible criteria (Buus et al., 1982). While a positive result is typical in cases of cochlear impairment, a negative result is found in most normal-hearing listeners and otherwise usually associated with retrocochlear disorders (Gelfand, 2001b). However, one should keep in mind that SISI
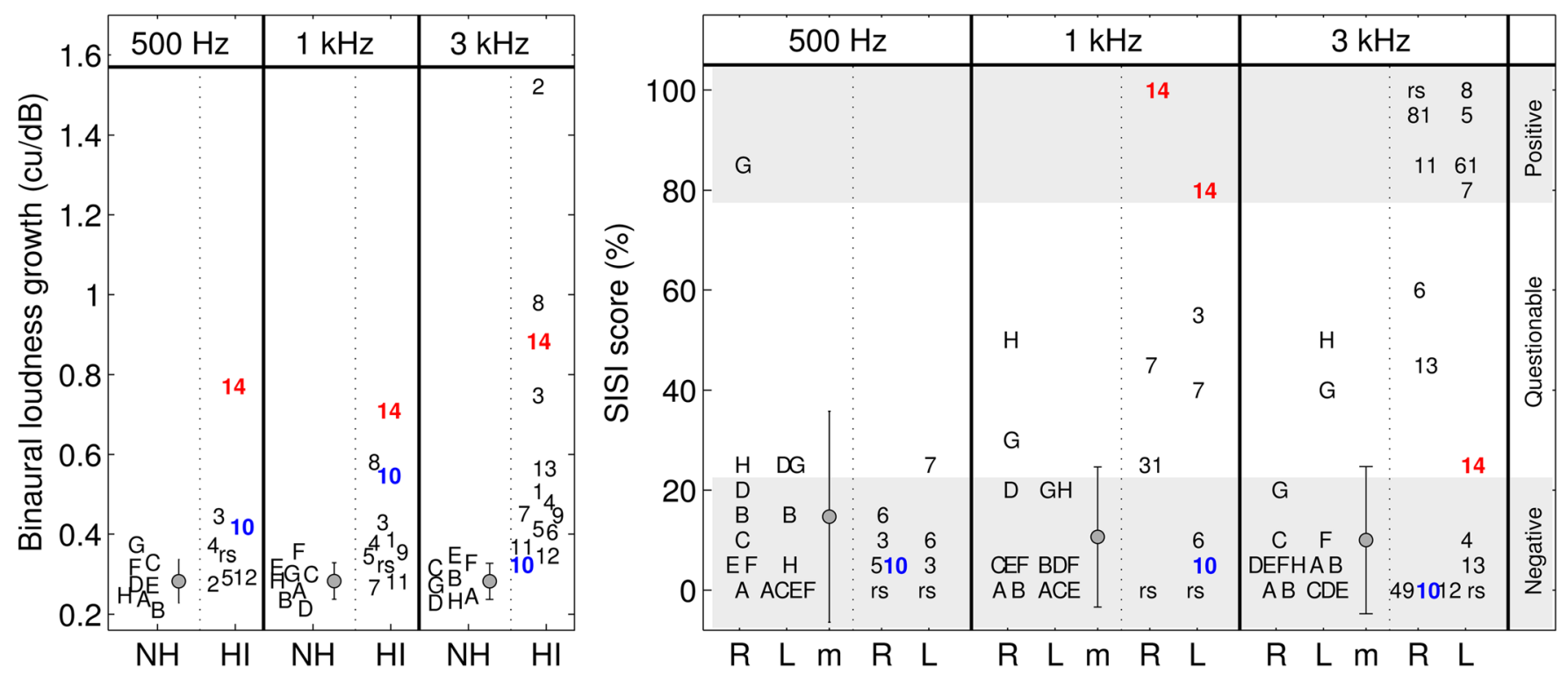

FIG. 3. (Color online) Slope of the lower section of the binaural loudness curve at 0.5 , 1, and $3 \mathrm{kHz}$ (left panel) and SISI scores (right panel) for NH and HI subjects. Individual results for the right (R) and left (L) ear, and mean and standard deviation across NH subjects (m). "rs" stands for "remaining subjects." 
scores have a high predictive value for cochlear losses (91\%), but only moderate predictive value for retrocochlear losses (48\%) (Buus et al., 1982).

The SISI scores of subjects 10 and 14 confirm the discrepancy in their loudness growth estimates. Subject 14 obtained positive or questionable results, again suggesting a cochlear impairment, while subject 10 consistently obtained negative SISI scores at all test frequencies, which might indicate a retrocochlear hearing loss. As other listeners showed little loss of audibility at low frequencies, only their SISI scores at $3 \mathrm{kHz}$ may be informative. Positive scores in both ears were found in subjects $1,5,6,7$, and 8 , suggesting cochlear hearing loss. Negative scores in both ears were found in subjects 4, 9, and 12. As subject 12 suffered from obscure dysfunction and showed a very strong OAE response, this indicates normal OHC function. The remaining listeners showed asymmetric SISI scores. Subjects 2, 3, and 11 obtained a positive result in the right ear, and a negative result in the left ear. For subject 2, this is consistent with an asymmetry in the acoustic reflex pattern, and with a strong OAE response. For subject 11, the asymmetry might reflect a similar asymmetry in hearing thresholds. Finally, subject 13 obtained a questionable score in the right ear and a negative score in the left ear. Taken together with her strong OAE response, this suggests normal OHC function. Overall, the combined results from the different audiometric tests do not allow further conclusions about the site of impairment for each $\mathrm{HI}$ listener.

\section{COGNITIVE ABILITIES}

The ability of the listeners to detect tonal targets and process pitch sequences in background noise may be influenced by high-level factors linked to global processing speed of sensory stimuli, working memory processing and capacity, and decision making, as suggested by studies investigating binaural pitch perception in dyslexic listeners (Chait et al., 2007; Santurette et al., 2010). Moreover, there is evidence that reduced processing speed and working-memory deficits play a role in the difficulty of $\mathrm{HI}$ listeners to understand speech in adverse conditions (e.g., van Rooij et al., 1989; van Rooij and Plomp, 1990; Lunner, 2003; Foo et al., 2007). Therefore, these cognitive functions were evaluated in a lexical decision task (e.g., Baddeley et al., 1985) and a reading span test (e.g., Daneman and Carpenter, 1980; Lunner, 2003), in order to investigate a potential effect on performance in the binauralpitch and auditory-profile tests.

\section{A. Lexical access and decision making}

A lexical decision task similar to that used by Baddeley et al. (1985) was performed. The subjects' task was to evaluate words as real or non-existing words. The proportion of correct responses and response times were measured. The percentage of words correctly identified as real or non-existing was above $92 \%$ for all $\mathrm{NH}$ and $\mathrm{HI}$ subjects. The response times of the individual subjects are given in the left panel of Fig. 4. The difference in response times between the $\mathrm{NH}$ and HI groups was only borderline significant ( $p=0.045$, twosample $t$-test), and most HI subjects lied within one standard deviation of the $\mathrm{NH}$ mean, including subjects 10 and 14. No
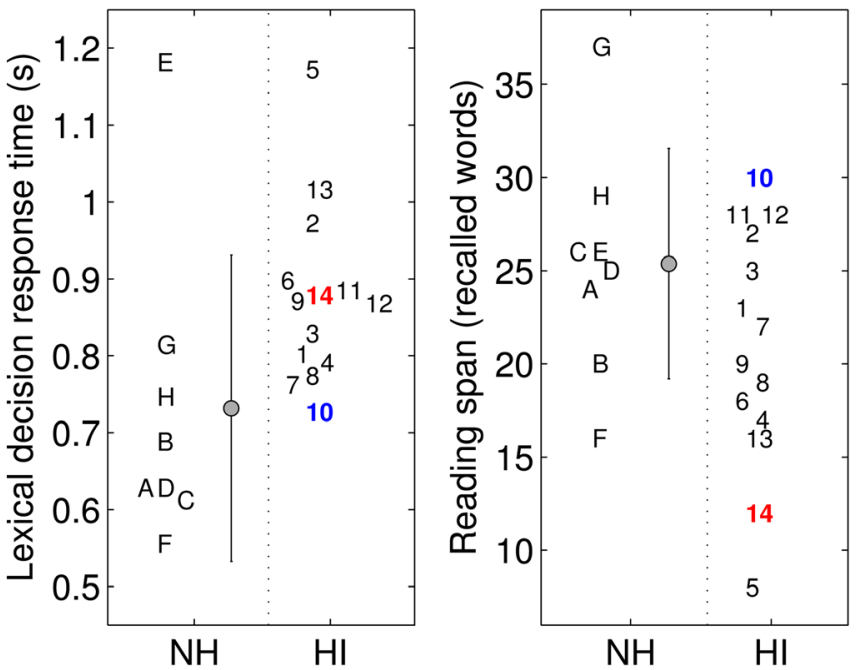

FIG. 4. (Color online) Response times in the lexical decision test (left panel) and reading span scores (right panel) for individual NH and HI subjects. The means and standard deviations across $\mathrm{NH}$ subjects are also given.

correlation was found between response times and either $\mathrm{MP}+\mathrm{BP}$ scores $(p=0.122)$ or MP $-\mathrm{BP}$ scores $(p=0.688)$.

\section{B. Working memory processing and capacity}

A reading span test similar to that used by Lunner (2003) was performed. The subjects' task was to evaluate sentences as normal or absurd, and to recall the first or last word in each sentence. The reading span was defined as the total number of correctly recalled words. The reading span scores are given in the right panel of Fig. 4. A large variability was found in both subject groups, and the range of reading span scores was similar to that obtained by Lunner (2003). There was no group difference between $\mathrm{NH}$ and $\mathrm{HI}$ subjects ( $p=0.130$, two-sample $t$-test). While subject 14 obtained a low reading span, subject 10 obtained the highest score among the HI subjects, indicating no influence of working memory on binaural pitch perception. Moreover, there was no correlation between reading span scores and either $\mathrm{MP}+\mathrm{BP}$ scores $(p=0.456)$ or MP - BP scores $(p=0.688)$.

\section{Discussion}

Overall, cognitive function, as measured by these two tests, was neither related to the ability to perceive binaural pitch, nor to the difference in identification scores between MP and BP stimuli. As a whole, performance in the cognitive tests was also not correlated with the ability of the listeners to perform the pitch contour identification task. However, subject 5 performed sensibly worse than all other HI subjects in both cognitive tasks, and subject 14 had a reading span below 15 words. As these two subjects were also those with the lowest MP + BP scores, an influence of reduced cognitive abilities on pitch contour identification cannot be completely excluded for these listeners. Interestingly, these were also the two listeners for whom pitch contour identification was affected by the use of noisebased stimuli. Finally, the fact that subject 10 was the best performer in both cognitive tests 
clearly indicates that the absence of binaural pitch percept does not imply reduced cognitive function.

\section{BINAURAL MASKING RELEASE}

\section{A. Binaural masking level difference (BMLD)}

The masking thresholds of $500-\mathrm{Hz}$ and $1000-\mathrm{Hz}$ tones in background noise were measured binaurally in two conditions. In the first condition, both the signal and the noise were diotic $\left(\mathrm{N}_{0} \mathrm{~S}_{0}\right.$ condition). In the second condition, the noise was diotic and an interaural phase shift of $180^{\circ}$ was introduced in the signal $\left(\mathrm{N}_{0} \mathrm{~S}_{\pi}\right.$ condition). For a given tone frequency, the BMLD was calculated as the difference in threshold between the $\mathrm{N}_{0} \mathrm{~S}_{0}$ and the $\mathrm{N}_{0} \mathrm{~S}_{\pi}$ condition.

Figure 5 shows the masked thresholds obtained at $500 \mathrm{~Hz}$ and $1 \mathrm{kHz}$ for the $\mathrm{N}_{0} \mathrm{~S}_{0}$ and $\mathrm{N}_{0} \mathrm{~S}_{\pi}$ conditions (left panel), as well as the resulting BMLDs at $500 \mathrm{~Hz}$ and $1 \mathrm{kHz}$ and the mean values for the two frequencies (right panel). With the exception of subject 14 at $500 \mathrm{~Hz}$, masked thresholds were always lower in the $\mathrm{N}_{0} \mathrm{~S}_{\pi}$ than in the $\mathrm{N}_{0} \mathrm{~S}_{0}$ conditions, indicating a release from masking for all listeners with the dichotic tone. The SNRs at threshold were significantly higher in the HI group than in the $\mathrm{NH}$ group for all conditions $\left(500 \mathrm{~Hz}-\mathrm{N}_{0} \mathrm{~S}_{0}: p=0.002\right.$, $500 \mathrm{~Hz}-\mathrm{N}_{0} \mathrm{~S}_{\pi}: p=0.037,1 \mathrm{kHz}-\mathrm{N}_{0} \mathrm{~S}_{0}: p=0.001,1 \mathrm{kHz}-\mathrm{N}_{0} \mathrm{~S}_{\pi}$ : $p=0.006$, two-sample $t$-tests). However, the group difference was not significant for the resulting BMLDs $(500 \mathrm{~Hz}$ : $p=0.276,1 \mathrm{kHz}: p=0.066$, mean: $p=0.120$, two-sample $t$-tests), and most HI listeners obtained BMLDs within the NH range. This is consistent with earlier reports of BMLDs (Staffel et al., 1990; Gabriel et al., 1992; Strelcyk and Dau, 2009), in which HI listeners showed elevated thresholds in both the diotic and dichotic conditions, leading to less pronounced differences with $\mathrm{NH}$ listeners in terms of masking release.

The two listeners with absent binaural pitch perception (10 and 14) obtained both elevated masked thresholds and a largely reduced masking release. Interestingly, the two subjects with the largest MP - BP scores (9 and 11) were also among the listeners with both the highest masked thresholds and the lowest BMLDs. This indicates that impaired binaural pitch perception coexists with a loss of binaural advantage in background noise. However, the latter does not imply the former (cf. subject 1). Subject 12 obtained the highest BMLDs among all listeners, including those from the NH group, confirming the findings of Strelcyk and Dau (2009) that listeners with obscure dysfunction do not show a deficit in binaural masking release.

Overall, there was no correlation in the whole HI group between mean BMLDs and either MP - BP scores $(p=0.307)$ or $\mathrm{MP}+\mathrm{BP}$ scores $(p=0.445)$. The mean BMLD was found to be significantly correlated with the asymmetry in hearing threshold ( $p=0.013, \rho=-0.65)$, suggesting that using the same presentation level in both ears might affect binaural masking release in cases of asymmetric hearing loss. BMLDs were also significantly correlated with mean OAE SNRs ( $p=0.006, \rho=0.71)$, as well as hearing thresholds at $500 \mathrm{~Hz}$ $(p=0.003, \rho=-0.73)$, but not at $1 \mathrm{kHz}(p=0.258)$. Finally, the correlation of BMLDs with dichotic masked thresholds was highly significant $(500 \mathrm{~Hz}: p<0.001,1 \mathrm{kHz}: p<0.001)$, indicating that performance in the $\mathrm{N}_{0} \mathrm{~S}_{\pi}$ condition is sufficient to predict the amount of masking release.

\section{B. Binaural intelligibility level difference (BILD)}

The subjects' SRT was measured in five different conditions, using Danish closed-set sentences (DANTALE II, Wagener et al., 2003). In the reference condition, both the speech signal and the masking noise were located in front of the listener and presented binaurally $\left(\mathrm{bS}_{0} \mathrm{~N}_{0}\right)$. In all other conditions, the target speech was kept in front of the listener, while the noise interferer was located on one side, with an azimuthal angle of $105^{\circ}$, where the largest amount of spatial release from masking is obtained (Peissig and Kollmeier, 1997). In two of the conditions, the speech and noise were
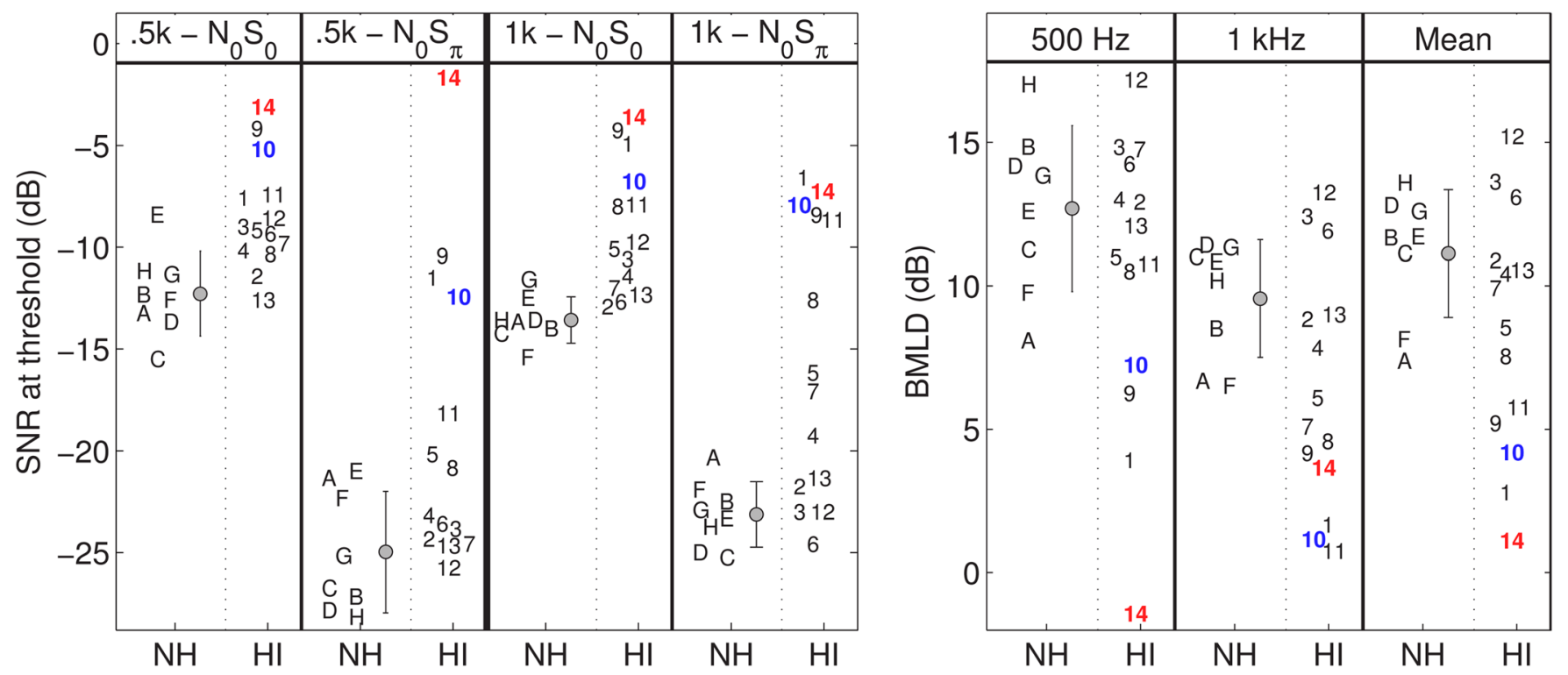

FIG. 5. (Color online) Binaural masked thresholds at 0.5 and $1 \mathrm{kHz}$ in $\mathrm{N}_{0} \mathrm{~S}_{0}$ and $\mathrm{N}_{0} \mathrm{~S}_{\pi}$ conditions (left panel) and resulting binaural masking level differences (right panel) for individual NH and HI subjects. The means and standard deviations across NH subjects are also given, as well as the mean BMLD across the two test frequencies. 
presented binaurally, with the noise located either on the right $\left(\mathrm{bS}_{0} \mathrm{~N}_{105}\right)$ or the left $\left(\mathrm{bS}_{0} \mathrm{~N}_{255}\right)$ side of the head. In the remaining two conditions, the speech and noise were presented monaurally to the ear opposite to the noise location, by setting the sound card attenuation to infinity in the right $\left(\mathrm{mS}_{0} \mathrm{~N}_{105}\right)$ or left $\left(\mathrm{mS}_{0} \mathrm{~N}_{255}\right)$ channel. The intelligibility level difference (ILD) was defined as the total amount of spatial release when the noise interferer was moved to the side:

(1) $\mathrm{ILD}_{\text {right }}=\operatorname{SRT}\left(\mathrm{bS}_{0} \mathrm{~N}_{0}\right)-\mathrm{SRT}\left(\mathrm{bS}_{0} \mathrm{~N}_{105}\right)$;

(2) ILD $_{\text {left }}=\operatorname{SRT}\left(\mathrm{bS}_{0} \mathrm{~N}_{0}\right)-\mathrm{SRT}\left(\mathrm{bS}_{0} \mathrm{~N}_{255}\right)$.

The binaural intelligibility level difference (BILD) was defined as the contribution of binaural interaction to the spatial release, i.e., the amount of spatial release not due to better-ear listening. It can be expressed as the difference in spatial release (or SRT) between the binaural and monaural conditions:

(1) $\mathrm{BILD}_{\text {right }}=\mathrm{SRT}\left(\mathrm{bS}_{0} \mathrm{~N}_{105}\right)-\mathrm{SRT}\left(\mathrm{mS}_{0} \mathrm{~N}_{105}\right)$;

(2) $\mathrm{BILD}_{\text {left }}=\mathrm{SRT}\left(\mathrm{bS}_{0} \mathrm{~N}_{255}\right)-\mathrm{SRT}\left(\mathrm{mS}_{0} \mathrm{~N}_{255}\right)$.

In the left panel of Fig. 6, the reference SRTs (obtained binaurally with the interfering noise at $0^{\circ}$ azimuth) are given for each individual subject. In the right panel, the total drop in SRT can be seen when the noise is moved to the right ( $\left.\mathrm{ILD}_{\text {right }}\right)$ or to the left ( $\mathrm{ILD}_{\text {left }}$ ), and is also represented as the average spatial release over these two conditions. The respective contributions of binaural processing to this spatial release (BILD) are then given.

The reference SRTs provide a measure of speech intelligibility in noise. All HI listeners except subject 3 obtained elevated SRTs compared to NH listeners, and the difference between the two groups was significant $(p<0.001$, twosample $t$-test). A significant correlation was found between SRTs and MP + BP scores $(p=0.009, \rho=-0.67)$. As SRTs were not correlated with pure-tone pitch identification scores $(p=0.415)$, this reflects the difficulty of some HI listeners in identifying signals in the presence of background noise, whether these signals are words or musical melodies. Significant correlations were also found between SRTs and $\mathrm{N}_{0} \mathrm{~S}_{\pi}$ masked thresholds $(500 \mathrm{~Hz}: p=0.014, \rho=0.64 ; 1 \mathrm{kHz}: p=$ $0.035, \rho=0.57)$, and thereby BMLDs $(500 \mathrm{~Hz}: p=0.006$, $\rho=-0.70 ; 1 \mathrm{kHz}: p=0.037, \rho=-0.56)$. Cognitive abilities have been shown to play an important role for speech reception in background noise [see Akeroyd (2008) for a review], and especially the reading span of HI listeners was found to correlate with measures of speech intelligibility in noise (e.g., Lunner, 2003; Foo et al., 2007). Neither the lexicaldecision response times $(p=0.441)$, nor the reading span scores $(p=0.463, p=0.629$ with age and audibility controlled for), were correlated with the reference SRTs in the present study. However, this may not be incompatible with the results of previous studies, and might just reflect the low statistical power of the present study due to a limited number of test subjects. No correlation was found between SRTs and MP - BP scores $(p=0.102)$, and there was a correlation of SRTs with low-frequency hearing thresholds $(p=0.021$, $\rho=0.61)$. Subject 12 obtained an elevated SRT compared to all NH listeners, despite a normal audiogram.

The total amount of spatial release when the interfering noise was moved to the side (ILD) was found to be significantly higher in NH than in HI listeners $\left(\operatorname{ILD}_{\text {mean }}: p=0.019\right.$, two-sample $t$-test), which is consistent with the findings of Peissig and Kollmeier (1997). However, in the present study, a significant correlation was found between mean ILDs and low-frequency hearing thresholds $(p<0.001, \rho=-0.79)$. Another significant, although borderline, correlation was found between mean ILDs and reference SRTs $(p=0.046$, $\rho=-0.54)$. Despite the significant group difference, most HI listeners obtained ILD $_{\text {mean }}$ values similar to those of $\mathrm{NH}$ listeners. Subjects 2 and 3 showed a slight decrease in ILD, while subjects 8,10 , and 14 had a considerably reduced spatial release from masking.
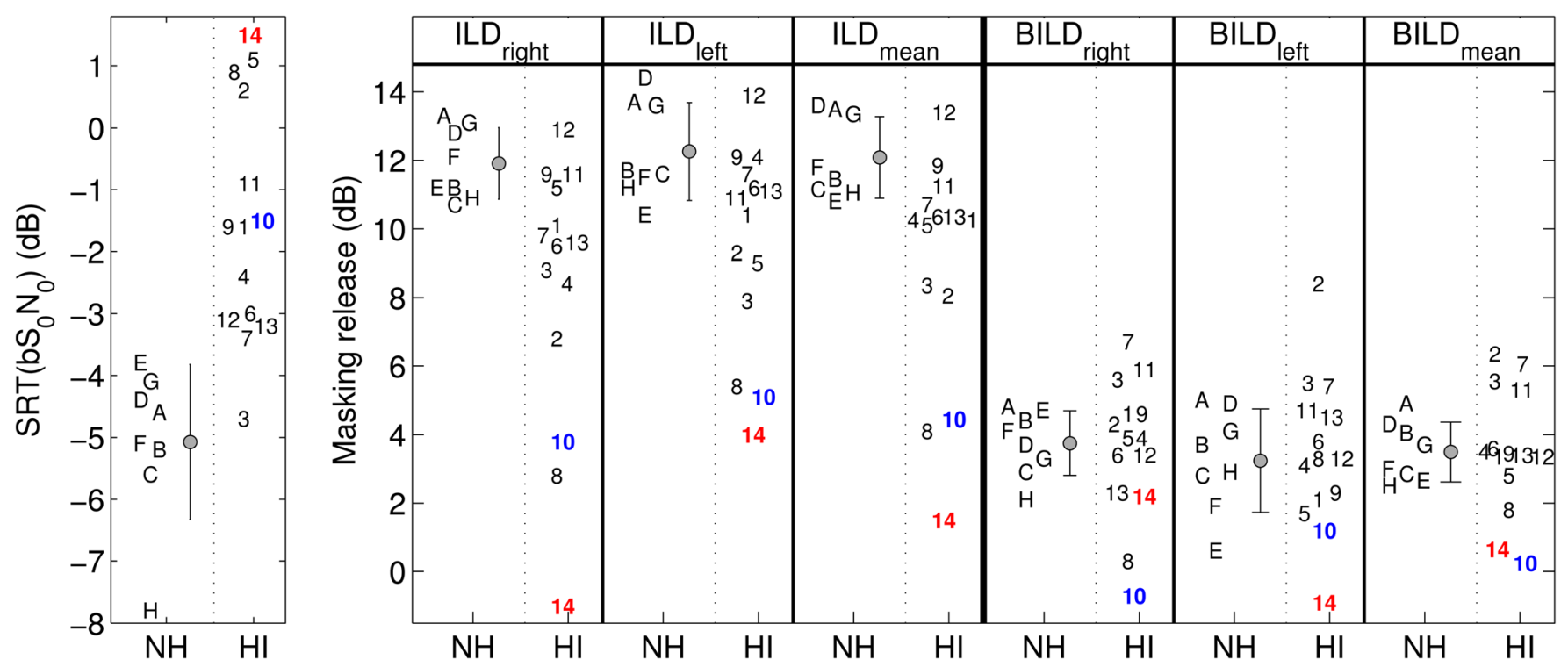

FIG. 6. (Color online) Speech reception thresholds in the $\mathrm{bN}_{0} \mathrm{~S}_{0}$ condition (left panel) and binaural intelligibility level differences (right panel) for individual $\mathrm{NH}$ and HI subjects. In the right panel, both the total amount of masking release (ILD) and the estimated binaural contribution (BILD) are given for the right and left ear. The means and standard deviations across NH subjects are also given, as well as the mean ILD and BILD across ears. 
Binaural processing was found to account for about $29 \%$ of the total spatial release in NH listeners, with a mean BILD of $3.5 \mathrm{~dB}$. This is a slightly lower binaural advantage than the ones reported in the review of Blauert (1997) and in more recent studies (Johansson and Arlinger, 2002; Goverts and Houtgast, 2010). However, most of these studies used experimental designs in which SRTs were compared for homophasic vs antiphasic speech or noise, and the use of a spatial design with head-related transfer functions (HRTFs) may be responsible for the different outcome in the present study. Overall, no group difference was found between $\mathrm{NH}$ and HI listeners for the mean BILD ( $p=0.961$, two-sample $t$-test). This indicates that all $\mathrm{HI}$ listeners who obtained mean ILDs above $8 \mathrm{~dB}$ showed some advantageous contribution of binaural processing to the spatial release. The two listeners who could not hear binaural pitch showed no binaural advantage in spatially segregating speech from noise. Mean BILDs were not correlated with $\mathrm{MP}+\mathrm{BP}$ scores $(p=0.929)$, MP $-\mathrm{BP}$ scores $(p=0.720)$, or low-frequency hearing thresholds $(p=0.053)$. A significant correlation was found between mean BILDs and mean BMLDs $(p=0.045, \rho=0.54)$, as well as with BMLDs at $500 \mathrm{~Hz}(p=0.027, \rho=0.59)$ but not at $1 \mathrm{kHz}(p=0.205)$.

\section{TEMPORAL FINE STRUCTURE PROCESSING}

The ability of the listeners to use TFS cues was evaluated binaurally in an IPD detection task, in which the upper frequency limit for detecting a $180^{\circ}$ phase shift in a tone was measured. The carrier frequency was chosen as the tracking variable in order to investigate the effect of sensorineural hearing loss on the upper frequency limit of binaural phase locking. Moreover, as the maximum IPD in the BP stimulus was $180^{\circ}$, the inability of a listener to detect such a large IPD within the most salient range of $\mathrm{BP}$ would provide a clear explanation for the absence of binaural pitch sensation. As the IPD detection task may rely on both peripheral TFS processing and the integration and processing of binaural information, frequency-modulation detection thresholds (FMDTs) at a 2-Hz FM-rate were additionally used as a monaural measure. This is because FM detection at low FMrates is thought to primarily rely on accurate TFS processing (Moore and Sęk, 1996; Lacher-Fougère and Demany, 1998; Strelcyk and Dau, 2009). Only six of the NH subjects were available to participate in these experiments.

\section{A. Interaural phase difference detection}

The upper frequency threshold for detectability of a $180^{\circ}$ interaural phase difference (IPD) was measured. The results are given in the left panel of Fig. 7. NH listeners were able to detect the IPD up to a carrier-frequency of $1336 \mathrm{~Hz}$ on average. This value and the range of obtained thresholds are in line with the results of Ross et al. (2007b), obtained via a similar behavioral method as well as cortical auditory evoked magnetic responses to IPD changes. The fact that subject E's threshold $(899 \mathrm{~Hz})$ was lower than those of all other NH listeners, who all lay above $1250 \mathrm{~Hz}$, may reflect her age difference with the rest of the NH group. This is consistent with the significant decrease in thresholds found by Ross et al. (2007a) between young and middle-aged subjects.

A significant group difference was found between $\mathrm{NH}$ and HI listeners ( $p=0.026$, two sample $t$-test). Despite this, most HI listeners' thresholds were found to lie around $1000 \mathrm{~Hz}$. For such listeners, there is thus no apparent deficit in detection of a $180^{\circ} \mathrm{IPD}$, given the age difference between the NH and HI groups. Subjects 10 and 14, however, obtained dramatically lower thresholds than all other HI listeners (349 and $210 \mathrm{~Hz}$, respectively). Therefore, there is a specific deficit for these two listeners in using interaural phase cues, even in quiet, which cannot be accounted for by an age factor. Furthermore, thresholds in the HI group were not correlated with age $(p=0.368)$. As binaural pitch
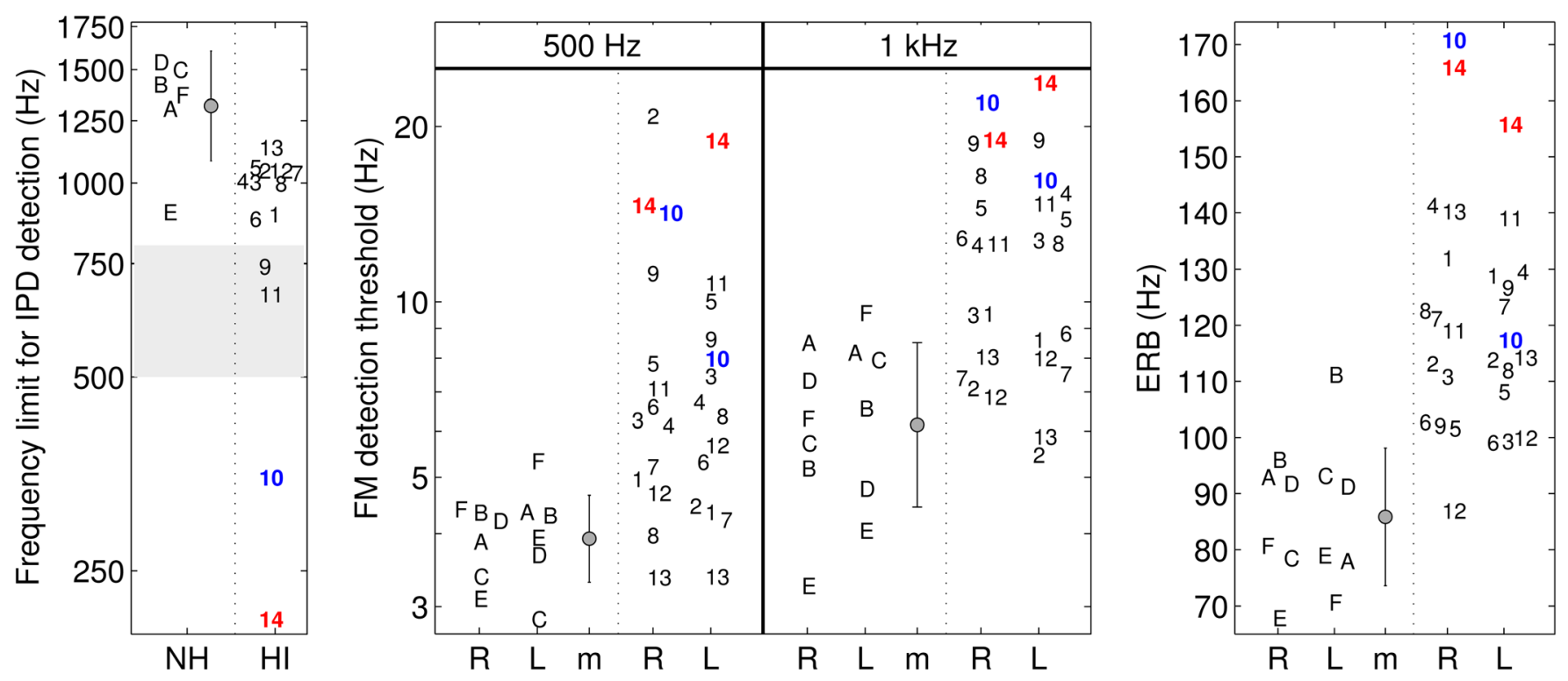

FIG. 7. (Color online) Upper frequency limit for detection of a $180^{\circ}$ interaural phase difference (left panel), frequency modulation detection thresholds at 0.5 and $1 \mathrm{kHz}$ (center panel), and ERB of the auditory filter estimates at $500 \mathrm{~Hz}$ (right panel) for $\mathrm{NH}$ and $\mathrm{HI}$ subjects. Individual results for the right (R) and left (L) ear, and mean and standard deviation across NH ears (m). In the left panel, the gray area indicates the frequency range of the notes used in the pitch contour identification experiment. 
perception relies on the introduction of an IPD in a noise stimulus, and as the note frequencies used in the binaural pitch experiments were all above $500 \mathrm{~Hz}$, the inability of subjects 10 and 14 to detect IPDs at such frequencies explains why they could not perceive any pitch in the BP stimulus. Interestingly, subjects 9 and 11, who had the highest MP - BP scores, obtained thresholds within the frequency range of the notes used in the pitch contour identification experiment (gray area in the left panel of Fig. 7). This suggests that they may not have heard the higher notes when played with the BP stimulus, leading to higher MP - BP scores.

IPD detection thresholds were found to be significantly correlated with BMLDs at $500 \mathrm{~Hz}(p=0.025, \rho=0.59)$ and $1 \mathrm{kHz}(p=0.009, \rho=0.67)$, consistent with the fact that both tasks involve the use of accurate interaural timing representations for detecting either the presence of a tone in noise (BMLD) or a change in the spatiality of a sound image (IPD detection). Following the correlation of BMLDs with OAE SNRs, IPD detection thresholds were also correlated with the mean OAE SNRs of the listeners $(p=0.050$, $\rho=0.53)$. No correlation was found between IPD detection thresholds and either $\mathrm{MP}+\mathrm{BP}(p=0.315)$ or $\mathrm{MP}-\mathrm{BP}$ $(p=0.508)$ scores. The correlation of IPD detection thresholds with low-frequency hearing thresholds was borderline significant ( $p=0.046, \rho=-0.54)$. This raises the questions of whether the observed deficits are suprathreshold deficits or a direct consequence of a loss of audibility, and whether there was an effect of using different sensation levels across subjects on the obtained thresholds. Using IPD detection tasks in which the IPD was the tracking variable, LacherFougère and Demany (2005) found no effect of sensation level on performance, and Strelcyk and Dau (2009) obtained thresholds that were not correlated with audibility. These findings, together with the fact that stimuli were adjusted for equal loudness and sensation levels never fell below $20 \mathrm{~dB}$ SL in the present study, strongly suggest that factors other than audibility were responsible for the observed deficits in binaural TFS processing.

\section{B. Frequency modulation detection}

Pure-tone FMDTs were measured in quiet for the subjects' left and right ear at 500 and $1000 \mathrm{~Hz}$. The obtained FMDTs are shown in the center panel of Fig. 7. The mean FMDTs over NH ears were $3.97 \mathrm{~Hz}(0.79 \%)$ at $500 \mathrm{~Hz}$ and $6.44 \mathrm{~Hz}(0.64 \%)$ at $1 \mathrm{kHz}$. These values are slightly higher than those reported by Grant (1987) and Demany and Semal (1989) at the same frequencies, and consistent with FMDTs obtained at other frequencies by Strelcyk and Dau (2009), whose experimental procedure was used in the present study. In line with these three studies, there was a significant increase in absolute FMDT (or decrease if expressed as a percentage) with frequency in both groups of listeners $(\mathrm{NH}$ : $p=0.031$, HI: $p<0.001$, Wilcoxon paired signed-rank test).

With the exception of HI subjects 1, 7, 12, and 13, HI listeners showed elevated FMDTs, suggesting a deficit in monaural TFS processing. The group difference between $\mathrm{NH}$ and $\mathrm{HI}$ listeners was significant at both frequencies
$(500 \mathrm{~Hz}: p=0.005,1 \mathrm{kHz}: p=0.003)$, confirming the adverse effects of sensorineural hearing loss on low-rate FM detection found in earlier studies (e.g., Lacher-Fougère and Demany, 1998; Moore and Skrodzka, 2002). Subjects 10 and 14 were among the HI listeners with the highest FMDTs at both test frequencies, and so were subjects 9 and 11, indicating that their deficit in TFS processing does not only reflect a specific binaural impairment. Moreover, mean FMDTs at $500 \mathrm{~Hz}$ were significantly correlated with MP - BP scores $(p=0.014, \rho=0.69)$, further suggesting that impaired binaural-pitch perception mainly stems from a poor peripheral representation of fine temporal information. The correlation between FMDTs at $1 \mathrm{kHz}$ and MP - BP scores was not significant $(p=0.130)$, probably reflecting the fact that note frequencies in the pitch contour identification experiment did not exceed $800 \mathrm{~Hz}$. Despite the above correlation, a clear-cut relationship between binaural-pitch perception and accurate monaural TFS processing cannot be established, as some listeners with highly elevated FMDTs did not show impaired binaural-pitch perception (subjects 2 and 5 at $500 \mathrm{~Hz}$, subjects 4,5 , and 8 at $1 \mathrm{kHz}$ ). Moreover, the assumption that FM-detection at low rates exclusively relies on TFS processing may not fully hold. More central deficits unrelated to TFS processing may indeed play a role in the elevated FMDTs observed in some listeners (LacherFougère and Demany, 1998), which is also suggested by the significant correlation of FMDTs at $500 \mathrm{~Hz}$ with MP + BP $\operatorname{scores}^{3}(p=0.002, \rho=-0.75)$.

The asymmetry in the accuracy of peripheral TFS representation between ears is another factor which may affect binaural-pitch perception. The relative asymmetry of individual FMDTs, expressed as the difference in thresholds divided by the mean left and right FMDT, is given in Table I (column "FM-As"). Subject 10 obtained a particularly high asymmetry in FMDT. However, other listeners with highly asymmetric FMDTs were able to perceive binaural pitch (cf. subjects 2 and 8 ). Moreover, MP - BP scores were not correlated with FMDT asymmetry $(500 \mathrm{~Hz}: p=0.194,1 \mathrm{kHz}$ : $p=0.470$ ) and there was no group difference in FMDT asymmetry between NH and HI listeners $(p=0.627)$, suggesting that such asymmetries only have a limited or no effect on binaural pitch perception.

A significant correlation was found between mean FMDTs and BMLDs at $1 \mathrm{kHz}(p=0.035, \rho=-0.57)$, but not at $500 \mathrm{~Hz}(p=0.192)$. However, the correlations of FMDTs with $\mathrm{N}_{0} \mathrm{~S}_{\pi}$ masked thresholds were significant at both frequencies $(500 \mathrm{~Hz}: p=0.038, \rho=0.56 ; 1 \mathrm{kHz}: p=0.021$, $\rho=0.61$ ). Consistent with this, FMDTs showed correlations with IPD detection thresholds which were significant at $1 \mathrm{kHz}$ $(p=0.006, \rho=-0.69)$ and only borderline at $500 \mathrm{~Hz}$ $(p=0.058, \rho=-0.52)$. This follows the observations of Strelcyk and Dau (2009), who found correlations between binaural and monaural measures of TFS processing in HI listeners. The correlation between FMDTs at $1 \mathrm{kHz}$ and mean BILDs was also significant ( $p=0.018, \rho=-0.62$ ).

Overall, binaural measures involving the use of interaural phase or time cues, both in noise and in quiet, may thus mainly rely on monaural TFS-processing skills. FMDTs at both frequencies were also correlated with reference SRTs 
$(500 \mathrm{~Hz}: p=0.035, \rho=0.57 ; 1 \mathrm{kHz}: p=0.046, \rho=0.54)$. This is in line with the results of Buss et al. (2004), who found a similar correlation, suggesting a role of TFS processing deficits in the reduced speech reception of listeners with sensorineural hearing loss. Finally, FMDTs were significantly correlated with hearing thresholds $(500 \mathrm{~Hz}: p=0.012$, $\rho=0.47$; $1 \mathrm{kHz}: p<0.001, \rho=0.67)$. This reflects the heterogeneity of the listeners in terms of their audiograms, and is in line with the significant correlation of FMDTs with hearing thresholds reported by Lacher-Fougère and Demany (1998). This does nevertheless not exclude the presence of suprathreshold deficits, as listeners with similar audiograms may exhibit widely different FMDTs (Strelcyk and Dau, 2009).

\section{FREQUENCY SELECTIVITY}

A notched-noise paradigm (Patterson and Nimmo-Smith, 1980; Patterson and Moore, 1986) was used to derive auditory filter shapes at $500 \mathrm{~Hz}$ in the subjects' left and right ears. Only six of the NH subjects participated in this experiment. The best-fitting rounded-exponential filter was estimated using the roex $\left(p_{u}, p_{l}, r\right)$ filter model (Patterson et al., 1982; Glasberg and Moore, 1990). The average rms fitting error over all ears was $0.67 \pm 0.24 \mathrm{~dB}$, indicating reasonable fits provided by the model. The estimated auditory-filter equivalent rectangular bandwidths (ERB) (Glasberg and Moore, 1990) for individual ears are given in the right panel of Fig. 7. The mean auditory filter bandwidth in the NH group was $85.9 \mathrm{~Hz}$. Considering the low number of subjects and the resulting standard deviation $(12.3 \mathrm{~Hz})$, this is in good agreement with the value of $78.7 \mathrm{~Hz}$ suggested by the formula of Glasberg and Moore (1990), as well as the results of Moore et al. (1990) who reported a mean value of $87 \mathrm{~Hz}$ at $400 \mathrm{~Hz}$. The ERB values were significantly higher in the HI than in the NH group $(p<0.001)$, by a factor of 1.4 on average. Subjects 10 and 14 both showed very broad filters. However, MP - BP scores were not correlated with mean ERB values $(p=0.397)$. This supports earlier suggestions that reduced frequency selectivity coexists with impaired binaural pitch perception, but cannot alone account for it (Santurette and Dau, 2007; Nitschmann et al., 2010). Mean ERB values were not correlated with MP + BP scores $(p=0.923)$.

The relative asymmetry between left and right ERB values, expressed as the difference in ERB divided by the mean left and right ERB, is given in Table I (column "ERB-As"). Most HI listeners did not show higher ERB asymmetry than $\mathrm{NH}$ listeners. Only subject 10 had an asymmetry factor more than double that of the NH group. This may be a contributing factor to her reduced performance in binaural TFS processing measures, and hence her inability to perceive binaural pitch. The outputs of left and right filters with different bandwidths may indeed show reduced correlation, leading to less effective binaural unmasking. However, subject 14 showed little asymmetry, and the lack of correlation between ERB asymmetry and MP $-\mathrm{BP}$ scores $(p=0.564)$, BMLDs $(p=0.062)$, and mean BILDs $(p=0.731)$, suggests that ERB asymmetry was not a crucial contributor to the observed deficits in binaural TFS processing.
The auditory filter bandwidths were significantly correlated with hearing thresholds at $500 \mathrm{~Hz}(p=0.029)$, confirming the relationship between audibility and frequency resolution found in earlier studies (e.g., Tyler et al., 1983; Strelcyk and Dau, 2009). However, the mean ERB values did neither correlate with speech reception $(p=0.329)$, mean BILDs $(p=0.239)$, IPD detection thresholds $(p=0.066)$, or FMDTs (500 Hz: $p=0.542,1 \mathrm{kHz}: p=0.128)$. The only other measure with which mean ERB values showed a significant correlation was the BMLD $(500 \mathrm{~Hz}: \quad p=0.017, \rho=-0.64 ; 1 \mathrm{kHz}$ : $p=0.005, \rho=-0.72$ ). Moreover, the examples of subjects 5 and 6 , who showed relatively narrow filters but elevated FMDTs, and of subjects 1, 7, and 13, who obtained normal FMDTs despite broader filters, illustrate that deficits in frequency selectivity and TFS processing do not necessarily covary. Therefore, the present findings are in line with those of Strelcyk and Dau (2009), providing further evidence that deficits in TFS processing cannot entirely be accounted for by poor frequency selectivity and reflect an additional impairment specific to the temporal acuity of internal sound representations.

\section{OVERALL SUMMARY AND DISCUSSION}

\section{A. Binaural pitch perception and the auditory profile}

Eight NH listeners and fourteen HI listeners with sensorineural hearing loss and various audiometric configurations performed a pitch contour identification task with binaural pitch stimuli and salience-matched monaurally detectable pitches. While most HI listeners could detect both pitch types as often as NH listeners, two of them were found not to perceive binaural pitch at all. Pitch contour identification scores showed that binaural pitch was clearly audible for all other listeners, but significantly less salient than the monaurally-detectable pitch. This indicates that the impaired mechanisms in sensorineural hearing loss affect pitch perception of noise-based pitch-evoking stimuli to a larger extent if pitch extraction requires binaural processing. The controlled pitch detection and contour identification task used here was found to be a more reliable test for the ability to hear binaural pitch than a short scale test (Santurette and Dau, 2007), in which a negative response may not imply absent binaural pitch perception.

The outcome of the binaural pitch experiments was compared to the listeners' performance in measures of several specific auditory and cognitive functions. The "BP" entry in Table III summarizes the relationship between binaural pitch perception and these auditory-profile measures. ${ }^{4}$ The results from a lexical decision task and a reading span test showed that cognitive abilities were unrelated to binaural pitch perception. However, deficits in binaural pitch perception coexisted with a large reduction in binaural release from masking, according to BMLD and BILD estimations. This nearly absent binaural advantage in detecting tones or understanding speech in background noise was linked to a more fundamental deficit in the processing of interaural phase information in quiet, illustrated by very low upperfrequency limits for detecting a large IPD. Such IPD detection thresholds were clearly related to the ability of the listeners to hear binaural pitch in the frequency range where it 
TABLE III. Summary of the relationship between outcomes of the different experiments. ++ : significant correlation. + : significant correlation for at least one test frequency. +b: reduced performance in listeners with impaired binaural pitch perception. - : no relationship found. BP refers to binaural pitch perception, " "Cog." to cognitive abilities, and "Aud." to audibility.

\begin{tabular}{lccccccccccc}
\hline \hline & BP & Cog. & SRT & ILD & BILD & BMLD & IPD & FM & ERB & Aud. \\
\hline BP & & - & - & $+\mathrm{b}$ & $+\mathrm{b}$ & $+\mathrm{b}$ & $+\mathrm{b}$ & + & - & - \\
Cog. & - & & - & - & - & - & - & - & - & - \\
SRT & - & - & & ++ & - & ++ & - & ++ & - & ++ \\
ILD & $+\mathrm{b}$ & - & ++ & & - & - & - & - & - & ++ \\
BILD & $+\mathrm{b}$ & - & - & - & & + & - & + & - & - \\
BMLD & $+\mathrm{b}$ & - & ++ & - & + & & ++ & + & ++ & + \\
IPD & $+\mathrm{b}$ & - & - & - & - & ++ & & + & - & ++ \\
FM & + & - & ++ & - & + & + & + & & - & ++ \\
ERB & - & - & - & - & - & ++ & - & - & & ++ \\
Aud. & - & - & ++ & ++ & - & + & ++ & ++ & ++ & \\
\hline \hline
\end{tabular}

is most salient. This suggests that accurate TFS processing up to the stage of binaural integration is a crucial factor for a binaural pitch sensation to arise. Although the listeners with absent binaural pitch perception exhibited broad auditory filters, reduced frequency selectivity did not account for the reduced salience of binaural pitch in HI listeners, suggesting a primary role of TFS processing.

At the output of the binaural processing stage, the accurate representation of precise timing information relies on two factors: a good peripheral temporal acuity as conveyed via phase-locking, and an accurate comparison of temporal inputs from the left and right channels via a well-functioning binaural processor. As both factors can affect performance in the IPD detection task, an additional monaural measure thought to primarily rely on peripheral phase-locked information, FM detection at a low FM-rate, was carried out. Here again, the listeners with absent binaural pitch perception performed markedly more poorly than other HI listeners, suggesting impaired peripheral representations of TFS in listeners unable to hear binaural pitch. A similar degree of monaural TFSprocessing deficit was, however, also found in some listeners with immediate binaural pitch perception, suggesting that the reduced acuity of phase-locked temporal information in the periphery is not a sufficient factor to make binaural pitch perception break down completely. Despite this, the significant correlation observed between 500-Hz FMDTs and the difference in pitch identification scores for MP vs BP stimuli supports the existence of a link between the acuity of peripheral TFS processing and the salience of binaural pitch.

In summary, binaural pitch perception was clearly found to primarily rely on TFS processing abilities, but it remains uncertain whether the peripheral or central mechanisms involved in processing fine temporal information are most crucial for binaural pitch extraction. Only a study on a large number of subjects with specific diagnoses might further reveal whether the absence of binaural pitch percept is a valid indicator of a particular auditory disorder.

\section{B. Correlations between the auditory profile measures}

Overall, the listeners from the HI group showed significant deficits in masked detection of homophasic and antipha- sic tones, speech intelligibility in background noise, spatial release from masking, binaural and monaural TFS processing, and frequency selectivity. While a subgroup of HI listeners showed reduced binaural advantage in noise, others could benefit from binaural processing to the same degree as $\mathrm{NH}$ listeners for detecting dichotic tones in noise or understanding speech in lateralized noise. Measures of lexical decision and working memory processing and capacity did not reveal highly significant differences between NH and HI listeners. Furthermore, cognitive abilities were not correlated with any of the measures included in the auditory profile. The only listener suffering from obscure dysfunction showed reduced speech reception in noise compared to NH listeners. However, no clear deficit was found for this listener in any of the other auditory-profile tests.

The study of correlations between the different auditory-profile measures, summarized in Table III, revealed an important role of monaural TFS processing ("FM" entry) for a variety of other tasks, including the ability to process interaural phase disparities both in quiet and in noise, but also to identify pitch contours and understand speech in background noise. The correlations between monaural TFS processing abilities and the performance in the binaural tasks do not support the presence of a specific binaural component in sensorineural hearing loss. This conclusion nevertheless relies on the assumption that elevated FMDTs exclusively reflect a monaural TFS deficit, which remains controversial (Lacher-Fougère and Demany, 1998). Moreover, the listeners who could detect a $180^{\circ}$ IPD might still have difficulties in detecting smaller IPDs. Consequently, good performance in the IPD detection experiment does not rule out the presence of additional deficits in binaural TFS processing. Asymmetries between ears in the different monaural measures were generally not found sufficient to account for reduced performance in binaural tasks, even though they might be a contributing factor.

Most notable was the absence of correlation between auditory filter bandwidths and TFS-related measures, suggesting that the TFS-processing deficits observed in HI listeners are at least partly independent of frequency selectivity. While the effect of a loss of OHCs on phase-locking precision remains uncertain, the loss of IHCs and auditory nerve fibers seem the most plausible factors likely to affect the peripheral representation of TFS information (Moore, 2007; Strelcyk and Dau, 2009). However, the extent to which damage in each of these entities contributes to impaired TFS processing remains difficult to quantify. Although the absence of correlation between TFS-processing measures and frequency selectivity is in agreement with the findings of, e.g., Strelcyk and Dau (2009) and Hopkins and Moore (2011), a cautionary note should be made concerning the small number of subjects used here and in previous studies. Small sample sizes impose strong limits to statistical power, and hence to the conclusions that can be drawn from not finding high correlations. The clarification of the relationship between frequency selectivity and TFS-processing abilities would thus benefit from additional studies on much larger groups of listeners, as a weak correlation between the two measures remains possible. 
The heterogeneity of the audiogram shapes in the HI group should also be mentioned as a possible confounder in the present study, as it resulted in the correlation of most measures with audibility ("Aud." entry in Table III). Particularly listeners with elevated low-frequency hearing thresholds often showed markedly poorer performance than most other HI listeners, leading to significant correlations with low-frequency audibility. Although the use of loudnessadjusted stimuli was aimed at limiting the influence of low sensation levels on the different outcomes, such an influence cannot be excluded here, as binaural performance is increasingly affected by presentation level as the latter approaches hearing threshold (e.g., Hershkowitz and Durlach, 1969). However, the presence of recent counterexamples indicates that audibility cannot alone account for a loss of binaural pitch percept (Nitschmann et al., 2010), and there is evidence of suprathreshold deficits in HI listeners, at least concerning TFS processing, from studies on homogeneous groups of listeners in terms of audibility (e.g., Strelcyk and Dau, 2009).

No clear distinction could be made between listeners with supposed cochlear vs retrocochlear hearing losses in any of the auditory-profile tests. While the lack of formal diagnoses and the difficulty of interpreting audiometric and loudness measures prevented a clear classification of the listeners in such subgroups, one should also keep in mind that each auditory-profile measure might be prone to several factors from different sites of impairment. The relative contribution of hair-cell vs nerve-cell loss on spectral and temporal resolution is an obvious example of this. If the need for reliable site-of-lesion tests persists, the present findings may help to define which basic features of hearing are primarily at stake and how they relate in cases of sensorineural hearing loss. Specifically, they underline the presence of TFS processing deficits which cannot be fully accounted for by a loss of frequency selectivity, and may adversely affect speech and pitch perception in background noise. Consequently, the evaluation of TFS processing abilities in HI patients would seem a valuable addition to audiometric measures and an informative tool in terms of general hearing abilities. In contrast, a measure of frequency selectivity may be redundant in a time-constrained context, due to the observed correlations with audibility, and may reflect the difficulties of HI listeners in other tasks to a lesser extent.

The present findings are thus in line with the increasing evidence for an important and independent role of TFS processing in hearing (e.g., Lacher-Fougère and Demany, 2005; Lorenzi et al., 2006; Hopkins et al., 2008; Strelcyk and Dau, 2009). However, this remains a controversial issue. One reason for this is the current lack of a reliable non-invasive measure of peripheral TFS processing in humans. Uncertainties persist concerning the role of a temporal mechanism for FM detection at very low rates (Lacher-Fougère and Demany, 1998), the measure chosen in the present study. Another disputed method is the discrimination of harmonic and frequency-shifted bandpass-filtered complex tones (Moore and Sęk, 2009), for which the sole role of TFS information has been questioned (Oxenham et al., 2009), and which cannot be used at low frequencies. The search for a psychophysical or physiological outcome that would accurately reflect peripheral TFS-processing abilities thus ought to be pursued. Until this is achieved, given the observed dependence of binaural TFS-processing outcomes on peripheral phase-locking acuity, the binaural IPD detection test used here may be a useful tool to quickly reveal deficits in the use of low-frequency TFS cues. The latter test proved a fast and reliable ${ }^{5}$ measure with a relatively simple task. This is unlike most auditory-profile tests used in the present study, which were well suited to a laboratory study on a few listeners but, with the exception of audiometric and loudnessperception measures, would have been too time-consuming in a clinical set-up. Therefore, further efforts to design an adequate battery of tests would be beneficial. Ideally, such a battery should allow an evaluation of each subject's auditory profile, in detail, but quickly, and without redundancy. Whether the inclusion of a binaural-pitch test would be a valuable addition to another measure of binaural TFS processing such as IPD detection could not be determined here. In this respect, the outcome of a short binaural-pitch test on a large population of listeners with confirmed specific sites of impairments would be very informative.

\section{ACKNOWLEDGMENTS}

The authors would like to thank the associate editor, Laurent Demany, as well as two anonymous reviewers for their constructive comments on earlier versions of this manuscript, Sarah Verhulst, for her help with setting up and analyzing OAE measurements, and Olaf Strelcyk, for providing the code used in the FM-detection and notched-noise experiments. The authors are also grateful to all participants for their motivation and patience throughout this study, and for accepting long hours of extensive testing. This work was supported by a DTU stipend.

\section{APPENDIX: METHODS FOR THE AUDITORY PROFILE TESTS}

\section{Pure-tone audiometry, tympanometry, acoustic reflex (Secs. IV A and IV B)}

Air-conduction thresholds were obtained in both ears at each of the following frequencies: 125, 250, 500, 1000, 2000, 3000, 4000, and $8000 \mathrm{~Hz}$. Bone-conduction thresholds were obtained in both ears at 500, 1000, 2000, and $4000 \mathrm{~Hz}$. All audiograms were measured using the Interacoustics AC440 audiometry module for the Affinity hearing-aid analyzer. An Interacoustics AT235 impedance audiometer was used to evaluate middle-ear function and acoustic reflexes.

\section{Otoacoustic emissions (Sec. IV C)}

The click stimuli were generated in MATLAB, sent to an RME FireFace 800 A/D-D/A converter via the PA-WAVPLAY software, and presented to the test subjects via an ER-2 probe at a rate of 20 clicks per second. The signal level of $70 \mathrm{~dB}$ peSPL was controlled with a DT-PA5 programmable attenuator. Recordings were made using an ER-10B lownoise microphone, and were bandpass filtered between 0.6 and $5 \mathrm{kHz}$ using an analog Rockland $852 \mathrm{HI} / \mathrm{LO}$ filter. The 
recorded analog signals were then converted and stored digitally, and the final click-response was defined as the average of 2000 recordings. Test subjects were instructed to lie down in a soundproof booth and keep still.

\section{Loudness scaling (Sec. V A)}

The stimuli were one-third octave bands of low-noise noise, geometrically centered at the test frequency. They were generated using method 1 as in Kohlrausch et al. (1997), and a stimulus duration of $1 \mathrm{~s}$ was used. The categorical scale contained 11 response alternatives (ISO 16832, 2006) and the subjects were instructed to click on one of the response bars after each presentation. Other test subject instructions, as well as the adaptive procedure used, were the same as in Brand and Hohmann (2002), except that the starting level and maximum level were $65 \mathrm{~dB}$ SPL and $115 \mathrm{~dB}$ SPL, respectively, and that three iterations were used in the second phase, with estimated levels $L_{10}, L_{20}, L_{30}$, and $L_{40}$ presented in the third iteration. After each block, the loudness function was obtained by fitting two independent linear functions to the lower $\left(L \leq L_{15}\right)$ and upper $\left(L \geq L_{35}\right)$ sections of the data. A Bézier smoothing was then used to link the two linear ends of the curve (Brand and Hohmann, 2002). When such a fit was not possible, the lower and upper sections of the curve were extended to $L \leq L_{20}$ and $L \geq L_{30}$, respectively, before the Bézier smoothing was applied. Further extensions of the linearly-fitted lower and upper ends of the curve, in steps of $5 \mathrm{cu}$, were used in some cases. If the variation in the subject's ratings was too large to obtain a loudness curve with this fitting method, the measurement was repeated.

\section{SISI test (Sec. V B)}

The automatic procedure of the Interacoustics AC222 audiometer was used. A continuous pure tone was presented at a level of $20 \mathrm{~dB}$ SL, and short intensity increments of 5, 2, or $1 \mathrm{~dB}$ occured at periodic time intervals. The task of the listeners was to press a response button every time an intensity increment was heard. The SISI score was calculated as the percentage of 1-dB increments correctly detected by the subject. Twenty 1-dB increments were presented for each condition.

\section{Cognitive tests (Sec. VI)}

Danish versions of both tests were implemented using the Psychophysics Toolbox extensions for MATLAB (Brainard, 1997; Pelli, 1997). Subjects were seated in front of a computer screen, such that the center of the screen was at eye level and the distance from their eyes to the screen was approximately $50 \mathrm{~cm}$. In both tests, words were presented in white bold capital letters on a dark green background at the center of the screen. Subjects were instructed to keep their attention to the center of the screen at all times, and a fixation point in the shape of a square appeared before each presentation. All room lights were switched off during testing.

\section{Lexical decision (Sec. VI A)}

Four lists containing 50 words each (25 real words and 25 non-words) were created, as well as a 10 -word practice list. All words contained 3 to 5 letters, and both started and ended with a consonant. Real words were monosyllabic adjectives or nouns, all situated between the 1000th and 2000th most frequent words in Danish (1998-2002), as selected from the Korpus 2000 database (DSL, 2002). Nonwords were nonhomophonic, phonologically different but visually similar to Danish language (pseudowords), and were obtained by modifying one letter from real Danish words. Each test list contained 9 three-letter, 12 four-letter, and 4 five-letter real words and non-words. Subjects gave their responses via a computer keyboard, and were instructed to place their right index on the " $\mathrm{K}$ " key, and their left index on the "F" key before the experiment started. Their task was to press "K" (korrekt/correct) as soon as a real word appeared on the screen and "F" (forkert/wrong) as soon as a non-word appeared. Before each word presentation, the fixation point was shown for a randomly chosen period of minimum 2 and maximum $4 \mathrm{~s}$. No feedback was provided. Each subject performed a single block on one test list, after one training session with the practice list. Words in each list were presented in a random order.

\section{Reading span (Sec. VI B)}

One list containing 54 four-word sentences (27 normal and 27 absurd) was created, as well as a 6-sentence practice list. Absurd sentences were all grammatically correct. Each sentence was presented word by word, and each word was visible for a fixed period of $800 \mathrm{~ms}$. After the last word of a sentence was shown, a question mark appeared at the center of the screen. In the same manner as in the lexical decision task, subjects were instructed to press " $\mathrm{K}$ " (korrekt/correct) if the sentence was normal and " $F$ " (forkert/wrong) if the sentence was absurd, as soon as the question mark appeared. Before each sentence presentation, the fixation point was shown for a randomly chosen period of minimum 2 and maximum $4 \mathrm{~s}$. No feedback was provided. After the presentation of a group of 3 to 6 sentences, the word "FØRST" (first) or "SIDST" (last) appeared on the screen, and the subjects were asked to verbally recall either the first or the last word of each sentence in the group. A voice-recording device was used to gather the subjects' responses. Subjects were allowed to guess and report words in any order, and had unlimited time to answer. The number of sentences in each group increased during the test, starting with three groups of 3 sentences, followed by three groups of 4,5 , and finally 6 sentences. First words had to be recalled for half of the groups, last words for the other half, and the sequence of "first" and "last" groups was randomized. Each subject performed a single test block, after one training session with the practice list. Sentences were presented in a random order.

\section{BMLD (Sec. VII A)}

In all conditions, the two-octave wide noise was geometrically centered around the test frequency. The noise level was fixed and equal to the binaurally-measured MCL at the test frequency. The signal level was varied adaptively in a three-interval, three-alternative forced-choice (3I$3 \mathrm{AFC}$ ) paradigm. Intervals had a 500-ms duration, including 
50-ms onset and offset cosine-ramps, and were separated by a 333-ms silent pause. A 1-up 2-down procedure was used, tracking the $70.7 \%$ point on the psychometric function (Levitt, 1971). For each presentation, one randomly chosen interval contained the tone signal in background noise, while the two other intervals contained noise only. The task of the subjects was to indicate via a computer keyboard which interval contained the tone. Feedback was provided. The starting signal-to-noise ratio (SNR) was $0 \mathrm{~dB}$. Stepsizes of 8, 3, and $1 \mathrm{~dB}$ were used, and the stepsize was decreased after each upper reversal. A block was terminated after ten reversals and the threshold value was determined from all points following the fourth reversal. Each subject performed three blocks with each condition, including one practice block. The final threshold was defined as the average threshold over the last two blocks.

\section{BILD (Sec. VII B)}

The interfering noise consisted of superimposed speech material from the DANTALE II sentences, yielding optimal spectral masking (Wagener et al., 2003). The spatial origin of the speech and noise stimuli was controlled virtually by convolving the waveforms with a set of HRTFs, as measured by Gardner and Martin (1994). Each subject was first introduced to the procedure and the speech material in a training block consisting of 30 sentences in the reference condition. A single test block of 20 sentences was then performed for each condition. In each block, the noise level was kept constant at the binaurally measured MCL at $500 \mathrm{~Hz}$, and the speech level was varied adaptively. Sentence lists were chosen randomly and the subjects' task was to verbally report the words in each sentence as they were understood, after each presentation. For each condition, the SRT was defined as the SNR for which $50 \%$ of individual words were correctly identified.

\section{IPD detection (Sec. VIII A)}

A 3I-3AFC procedure was used, in a paradigm similar to that of Ross et al. (2007b). Stimuli were sinusoidal-amplitude-modulated pure tones, with a $40-\mathrm{Hz}$ modulation rate and a modulation depth equal to 1 . The tracking variable was the frequency of the tone carrier. For each trial, three 750-ms intervals separated by 333-ms silent gaps were presented. In the two reference intervals, the left and right stimuli were in phase, and were perceived as a single sound source located inside the head. In the randomly chosen target interval, the left and right stimuli were in phase during the first half ( $375 \mathrm{~ms}$ ), and in antiphase during the second half of the interval, i.e., the sound was perceived as starting inside the head and suddenly becoming more spacious in the middle of the interval. As the modulation rate used corresponded to a 25-ms envelope period, the change in IPD always occured in a modulation dip, thus avoiding discontinuities in the waveform (Ross et al., 2007b). The task of the subjects was to indicate via a computer keyboard which interval contained the IPD change. Feedback was provided. A 2-up 1-down procedure was used to track the $70.7 \%$ point on the psychometric function (Levitt, 1971). The threshold frequency was tracked logarithmically, with an initial carrier frequency of $250 \mathrm{~Hz}$ and stepsizes of $1 / 2,1 / 5$, and 1/10 octave, which were decreased after each lower reversal. If a subject could not detect the target interval correctly in the first trial, the initial carrier frequency was reduced to $100 \mathrm{~Hz}$. A block was terminated after ten reversals and the threshold value was determined from all points following the fourth reversal. Each subject performed five test blocks and the final threshold was defined as the average threshold over all blocks. A presentation level of $50 \mathrm{~dB}$ SPL was used in NH subjects. In order to ensure sufficient audibility of the stimuli for HI listeners, the presentation level was adjusted to the higher of the binaurally-measured $L_{10}$ levels at 500 and $1000 \mathrm{~Hz}$ (Table I), when these were higher than $50 \mathrm{~dB}$ SPL. If the latter adjustment was insufficient to reach a level of $20 \mathrm{~dB}$ SL in both ears, the level corresponding to $20 \mathrm{~dB}$ $\mathrm{SL}$ in the worst ear was used. Although asymmetries in hearing threshold might disrupt the perceived location of the stimulus by introducing interaural level differences (ILDs), results from pilot testing showed that the IPD cue was still easily perceivable when an ILD of $15 \mathrm{~dB}$ or less was introduced. Moreover, an IPD of $180^{\circ}$ is much larger than the just noticeable difference in interaural phase for a $500-\mathrm{Hz}$ tone in NH listeners, for ILDs up to at least $20 \mathrm{~dB}$ (Hershkowitz and Durlach, 1969; Domnitz, 1973). Therefore, such an IPD is expected to be easily detectable in the presence of an ILD. Hearing threshold asymmetries were thus only compensated for by introducing an ILD in the stimulus when the mean asymmetry between 125 and $2000 \mathrm{~Hz}$ was equal to or larger than $15 \mathrm{~dB}$ (Table I).

\section{FM detection (Sec. VIII B)}

The stimuli and procedure were similar to those used by Strelcyk and Dau (2009). An FM-rate of $2 \mathrm{~Hz}$ and an FMphase of $1.5 \pi$ were used, such that the frequency-modulated stimulus could be described as

$$
x(t)=a(t) \sin \left[2 \pi f_{c} t+\frac{\Delta f}{2} \sin (\pi(4 t+1.5))\right],
$$

where $f_{c}$ is the carrier frequency and $\Delta f$ the maximum frequency excursion. Quasi-sinusoidal amplitude modulation (AM) was superimposed to the FM-tones in order to disrupt FM-to-AM conversion cues (Grant, 1987; Moore and Sęk, 1996), such that $a(t)$ was proportional to $1+m \sin (2 \pi F(t)+\Phi)$. The AM-depth $m$ was fixed at a peak-to-valley ratio of $6 \mathrm{~dB}$ and the AM-phase $\Phi$ was randomized. The integral of the instantaneous AM-rate was

$$
F(t)=\int_{0}^{t} d \tau\left(f_{1}+\frac{f_{2}-f_{1}}{T} \tau\right)
$$

where $T$ is the stimulus duration and $f_{1}$ and $f_{2}$ were randomly chosen between 1 and $3 \mathrm{~Hz}$ with $\left|f_{2}-f_{1}\right|>1 \mathrm{~Hz}$. An adaptive 3I-3AFC procedure was used, with an interval duration of $750 \mathrm{~ms}$, and $300 \mathrm{~ms}$ silent gaps between two successive intervals. All stimuli were gated with $50 \mathrm{~ms} \cos ^{2}$-ramps. In each trial, all three intervals were independently amplitude 
modulated while only the target interval was frequency modulated. The subjects' task was to indicate via a computer keyboard which interval contained the FM-tone. The $75 \%$ point on the psychometric function was tracked using a weighted up-down method (Kaernbach, 1991) in which $\Delta f$ was varied logarithmically. A block was terminated after 12 reversals and the threshold value was determined from all points following the fourth reversal. Each subject participated in a training session containing two blocks in each ear/ test-frequency condition. Three test blocks were then performed for each condition. Additional blocks were performed as long as the standard deviation over all blocks exceeded $15 \%$ of the mean FMDT, with a maximum of five blocks per subject. The final threshold was defined as the geometric mean over all blocks. The presentation order of the four different ear/test-frequency conditions was randomized for each subject. A $60 \mathrm{~dB}$ SPL presentation level was used, unless the subject's left or right $L_{10}$ level at $500 \mathrm{~Hz}$ or $1000 \mathrm{~Hz}$ was higher than $60 \mathrm{~dB}$ SPL, in which case the higher of the left and right monaurally measured $L_{10}$ at 500 and $1000 \mathrm{~Hz}$ was used (Table I). If the latter adjustment was insufficient to reach a level of $20 \mathrm{~dB}$ SL in both ears, the level corresponding to $20 \mathrm{~dB}$ SL in the worst ear was used.

\section{Frequency selectivity (Sec. IX)}

Target tones of 440-ms duration were temporally centered in 550-ms fixed-amplitude random-phase noise maskers, and $50 \mathrm{~ms} \cos ^{2}$-ramps were applied to both tones and maskers. The outside edges of the noise maskers were fixed at $\pm 0.8 f_{0}$, where $f_{0}$ is the signal frequency. Five symmetric $\quad\left(\Delta f / f_{0}=[0.0|0.0 ; 0.1| 0.1 ; 0.2|0.2 ; 0.3| 0.3 ; 0.4 \mid 0.4]\right)$ and two asymmetric $\left(\Delta f / f_{0}=[0.2|0.4 ; 0.4| 0.2]\right)$ notch conditions were measured, where $\Delta f$ is the spacing between $f_{0}$ and the inner noise edges. An adaptive 3I-3AFC weighted updown method (Kaernbach, 1991) was used, tracking the $75 \%$ point on the psychometric function. Successive intervals were separated by a $250 \mathrm{~ms}$ silent gap. In each trial, all three intervals contained the noise masker, while only the target interval contained the tone signal. The subjects' task was to indicate via a computer keyboard which interval contained the tone. The masker level varied adaptively while the signal level was kept constant at $50 \mathrm{~dB}$ SPL, unless the subject's left or right $L_{10}$ level at $500 \mathrm{~Hz}$ was higher than $50 \mathrm{~dB}$ SPL, in which case the monaurally measured $L_{10}$ in the worst ear was used (Table I). A block was terminated after 12 reversals and the threshold value was determined from all points following the fourth reversal. Each subject performed three test blocks in each condition, and the final threshold was defined as the average threshold over all blocks. The presentation order of the 14 different ear/notch conditions was randomized for each subject.

${ }^{1} L_{\mathrm{NBN}}=1.07 \times L_{\mathrm{BBN}}-15.69$, where $L_{\mathrm{BBN}}$ is the overall level of the broadband noise in both BP and MP stimuli and $L_{\mathrm{NBN}}$ is the overall level of the additional narrow band of noise in the MP stimulus.

${ }^{2} \mathrm{~A}$ total of 33 outcome measures were compared in this study, raising the issue of multiple testing. Among all possible comparisons, 105 correlation coefficients were used to interpret the relationship between the different outcomes. In order to find an appropriate significance level for this large set of correlations, a $p$-value plot (Schweder and Spjøtvoll, 1982) was drawn, and the marginal significance of the largest coefficients was calculated using the asymptotic theory of Buckley and Eagleson (1986). According to these two methods, a significance level $\alpha=5 \%$ was reasonable in the present context. Therefore, no correction was applied to the $p$ values reported in this paper.

${ }^{3}$ This correlation may at first suggest a role of TFS in pitch coding of MP stimuli as well as BP stimuli. However, one must keep in mind that the FM-detection task consisted in detecting the presence of a warble, which can be argued to involve a pitch cue. In that sense, it is possible that the FMDTs of some listeners, as well as the correlation of FMDTs with pitch contour identification scores, reflect a general ability of the listeners to follow pitch changes. Good performance in the FM-detection task might thus rely on more central pitch mechanisms, in addition to requiring accurate TFS processing.

${ }^{4}$ For the "BP" entry in Table III, "+" indicates a significant correlation of MP - BP scores with the corresponding measure at $500 \mathrm{~Hz}$, while "-_indicates the absence of any correlation between MP - BP scores and the corresponding measure. As MP - BP scores were not calculated for the two listeners who could not detect binaural pitch, " $+\mathrm{b}$ " is used to indicate that these subjects, as well as subjects with low MP - BP scores, showed considerably reduced performance in the corresponding tests.

${ }^{5}$ For the IPD-detection test, the within-subject standard deviation over all measurement blocks was on average $1 \%$ of the mean log threshold, indicating a good measurement repeatability. This is in contrast to the FM-detection test, for which the uncertainty around the individual thresholds is higher, with a median block standard deviation of $15 \%$ of the mean threshold.

Akeroyd, M. A. (2008). "Are individual differences in speech reception related to individual differences in cognitive ability? A survey of twenty experimental studies with normal and hearing-impaired adults," Int. J. Audiol. 47(S2), S125-S143.

Akeroyd, M. A., Moore, B., and Moore, G. A. (2001). "Melody recognition using three types of dichotic-pitch stimulus," J. Acoust. Soc. Am. 110(3), 1498-1504.

Al-Salim, S. C., Kopun, J. G., Neely, S. T., Jesteadt, W., Stiegemann, B., and Gorga, M. P. (2009). "Reliability of categorical loudness scaling and its relation to threshold," Ear Hear. 31(4), 567-578.

Baddeley, A., Logie, R., Nimmo-Smith, I., and Brereton, N. (1985). "Components of fluent reading," J. Mem. Lang. 24, 119-131.

Blauert, J. (1997). Spatial Hearing: The Psychophysics of Sound Localization (Massachusetts Institute of Technology, Cambridge), Chap. 3.

Brainard, D. H. (1997). "The psychophysics toolbox," Spatial Vision 10(4), 433-436.

Brand, T., and Hohmann, V. (2002). "An adaptive procedure for categorical loudness scaling.” J. Acoust. Soc. Am. 112(4), 1597-1604.

Buckley, M. J., and Eagleson, G. K. (1986). "Assessing large sets of rank correlations," Biometrika 73(1), 151-157.

Buss, E., Hall, J. W. III, and Grose, J. H. (2004). "Temporal fine-structure cues to speech and pure tone modulation in observers with sensorineural hearing loss," Ear Hear. 25(3), 242-250.

Buus, S., Florentine, M., and Redden, R. B. (1982). "The SISI test: A review." Audiology 21, 273-293.

Chait, M., Eden, G., Poeppel, D., Simon, J. Z., Hill, D. F., and Flowers, D. L. (2007). "Delayed detection of tonal targets in background noise in dyslexia," Brain Lang. 102, 80-90.

Cramer, E. M., and Huggins, W. H. (1958). "Creation of pitch through binaural interaction,” J. Acoust. Soc. Am. 30(5), 413-417.

Culling, J. F., Summerfield, A. Q., and Marshall, D. H. (1998). "Dichotic pitches as illusions of binaural unmasking. I. Huggins' pitch and the 'binaural edge pitch,"” J. Acoust. Soc. Am. 103(6), 3509-3526.

Daneman, M., Carpenter, P. A. (1980). "Individual differences in working memory and reading," J. Verb. Learn. Verb. Behavior 19, 450-466.

Demany, L., and Semal, C. (1989). "Detection thresholds for sinusoidal frequency modulation,” J. Acoust. Soc. Am. 85(3), 1295-1301.

Dix, M. R., Hallpike, C. S., and Hood, J. D. (1948). "Observations upon the loudness recruitment phenomenon, with especial reference to the differential diagnosis of disorders of the internal ear and VIII nerve," Proc. R. Soc. Med. 41, 516-526.

Domnitz, R. (1973). "The interaural time jnd as a simultaneous function of interaural time and interaural amplitude," J. Acoust. Soc. Am. 53(6), 1549-1552.

DSL (2002). Korpus 2000 - Dansk sprog omkring airtusindskiftet (The Danish language around year 2000) (Det Danske Sprogog Litteraturselskab, The Danish Language and Literature Association, Copenhagen). 
Durlach, N. I. (1960). "Note on the equalization and cancellation theory of binaural masking level differences," J. Acoust. Soc. Am. 32(8), 1075-1076.

Feldman, A. S. (1977). "Diagnostic application and interpretation of tympanometry and the acoustic reflex," Audiology 16, 294-306.

Foo, C., Rudner, M., Rönnberg, J., and Lunner, T. (2007). "Recognition of speech in noise with new hearing instrument compression release settings requires explicit cognitive storage and processing capacity," J. Am. Acad. Audiol. 18, 618-631.

Gabriel, K. J., Koehnke, J., and Colburn, H. S. (1992). "Frequency dependence of binaural performance in listeners with impaired binaural hearing," J. Acoust. Soc. Am. 91(1), 336-347.

Gardner, B., and Martin, K. (1994). "HRTF measurements of a KEMAR dummy-head microphone," Technical Report \#280 (Massachusetts Institute of Technology Media Lab Machine Perceptual Computing, Cambridge).

Gelfand, S. A. (2001a). "Acoustic immittance assessment," Essentials of Audiology, 2nd ed. (Thieme, New York), Chap. 7.

Gelfand, S. A. (2001b). "Behavioral tests for diagnostic assessment," Essentials of Audiology, 2nd ed. (Thieme, New York), Chap. 10.

Glasberg, B. R., and Moore, B. C. J. (1990). "Derivation of auditory filter shapes from notchednoise data," Hear. Res. 47, 103-138.

Goverts, S. T., and Houtgast, T. (2010). "The binaural intelligibility level difference in hearing impaired listeners: The role of supra-threshold deficits," J. Acoust. Soc. Am. 127(5), 3073-3084.

Grant, K. W. (1987). "Frequency modulation detection by normally hearing and profoundly hearing-impaired listeners," J. Speech Hear. Res. 30, 558-563.

Hershkowitz, R. M., Durlach, N. I. (1969). "Interaural time and amplitude jnds for a 500-Hz tone," J. Acoust. Soc. Am. 46(6), 1464-1467.

Hood, J. D. (1969). "Basic audiological requirements in neuro-otology," J. Laryngol. Otol. 83, 695-711.

Hopkins, K., Moore, B. C. J., and Stone, M. A. (2008). "The effects of moderate cochlear hearing loss on the ability to benefit from temporal fine structure information in speech," J. Acoust. Soc. Am. 123(2), $1140-1153$.

Hopkins, K., and Moore, B. C. J. (2011). "The effects of age and cochlear hearing loss on temporal fine structure sensitivity, frequency selectivity, and speech reception in noise," J. Acoust. Soc. Am. 130(1), 334-349.

ISO (2006). 16832:2006(E), Acoustics-Loudness Scaling by Means of Categories, Annex A (International Organization for Standardization, Geneva).

Jerger, J., Shedd, J. L., and Harford, E. (1959). "On the detection of extremely small changes in sound intensity," Archiv. Otolaryngol. 69(2), 200-211.

Jerger, J. (1970). "Clinical experience with impedance audiometry," Archiv. Otolaryngol. 92(4), 311-324.

Johansson, M. S. K., and Arlinger, S. D. (2002). "Binaural masking level difference for speech signals in noise," Int. J. Audiol. 41, 279-284.

Kaernbach, C. (1991). "Simple adaptive testing with the weighted up-down method," Percept. Psychophys. 49, 227-229.

King, K., and Stephens, D. (1992). "Auditory and psychological factors in auditory disability with normal hearing," Scand. Audiol. 21(2), 109-114.

Kohlrausch, A., Fassel, R., van der Heijden, M., Kortekaas, R., van de Par, S., Oxenham, A. J., and Püschel, D. (1997). "Detection of tones in lownoise noise: Further evidence for the role of envelope fluctuations," Acustica 83, 659-669.

Lacher-Fougère, S., and Demany, L. (1998). "Modulation detection by normal and hearing-impaired listeners," Audiology 37, 109-121.

Lacher-Fougère, S., and Demany, L. (2005). "Consequences of cochlear damage for the detection of interaural phase differences," J. Acoust. Soc. Am. 118(4), 2519-2526.

Levitt, H. (1971). "Transformed up-down methods in psychoacoustics," J. Acoust. Soc. Am. 49(2), 467-477.

Lorenzi, C., Gilbert, G., Carn, H., Garnier, S., and Moore, B. C. J. (2006). "Speech perception problems of the hearing impaired reflect inability to use temporal fine structure," Proc. Natl. Acad. Sci. USA 103(49), 18866-18869

Lunner, T. (2003). "Cognitive function in relation to hearing aid use," Int. J. Audiol. 42, S49-S58.

Moore, B. C. J. (2007). Cochlear Hearing Loss-Physiological, Psychological and Technical Issues, 2nd ed. (Wiley, Hoboken, NJ), Chap. 7.

Moore, B. C. J., Peters, R. W., and Glasberg, B. R. (1990). "Auditory filter shapes at low center frequencies," J. Acoust. Soc. Am. 88(1), 132-140.
Moore, B. C. J., Sẹk, A. (1996). "Detection of frequency modulation at low modulation rates: Evidence for a mechanism based on phase locking." J. Acoust. Soc. Am., 100(4, Pt. 1):2320-2331.

Moore, B. C. J., Sęk, A. (2009). "Development of a fast method for determining sensitivity to temporal fine structure," Int. J. Audiol. 48, 161-171.

Moore, B. C. J., and Skrodzka, E. (2002). "Detection of frequency modulation by hearing impaired listeners: Effects of carrier frequency, modulation rate, and added amplitude modulation," J. Acoust. Soc. Am. 111(1), 327-335.

Nitschmann, M., Verhey, J. L., and Kollmeier, B. (2010). "Monaural and binaural frequency selectivity in hearing-impaired subjects," Int. J. Audiol. 100(49), 357-367.

Oxenham, A. J., Micheyl, C., and Keebler, M. V. (2009). "Can temporal fine structure represent the fundamental frequency of unresolved harmonics?,” J. Acoust. Soc. Am. 125(4), 2189-2199.

Patterson, R. D., and Moore, B. C. J. (1986). "Auditory filters and excitation patterns as representations of frequency resolution," Frequency Selectivity in Hearing (Academic, London).

Patterson, R. D., and Nimmo-Smith, I. (1980). "Off-frequency listening and auditory-filter asymmetry,” J. Acoust. Soc. Am. 67, 229-245.

Patterson, R. D., Nimmo-Smith, I., Weber, D. L., and Milroy, R. (1982). "The deterioration of hearing with age: Frequency selectivity, the critical ratio, the audiogram, and speech threshold," J. Acoust. Soc. Am. 72, $1788-1803$.

Peissig, J., and Kollmeier, B. (1997). "Directivity of binaural noise reduction in spatial multiple noise-source arrangements for normal and impaired listeners," J. Acoust. Soc. Am. 101(3), 1660-1670.

Pelli, D. G. (1997). "The VideoToolbox software for visual psychophysics: Transforming numbers into movies," Spatial Vision 10(4), 437-442.

Priede, V. M., and Coles, R. R. A. (1974). "Interpretation of loudness recruitment tests," J. Laryngol. Otol. 88(2), 641-662.

Probst, R., Lonsbury-Martin, B. L., and Martin, G. K. (1991). "A review of otoacoustic emissions,” J. Acoust. Soc. Am. 89(5), 2027-2067.

Raatgever, J., and Bilsen, F. A. (1986). "A central spectrum theory of binaural processing. Evidence from dichotic pitch,” J. Acoust. Soc. Am. 80(2), 429-441.

Ross, B., Fujioka, T., Tremblay, K. L., and Picton, T. W. (2007a). "Aging in binaural hearing begins in mid-life: Evidence from cortical auditoryevoked responses to changes in interaural phase," J. Neurosci. 27(42), 11172-11178.

Ross, B., Tremblay, K. L., and Picton, T. (2007b). "Physiological detection of interaural phase differences," J. Acoust. Soc. Am. 121(2), 1017-1027.

Ruggero, M. A., and Rich, N. C. (1991). "Furosemide alters organ of Corti mechanics: Evidence for feedback of outer hair cells upon the basilar membrane," J. Neurosci. 11(4), 1057-1067.

Santurette, S., and Dau, T. (2007). "Binaural pitch perception in normalhearing and hearingimpaired listeners," Hear. Res. 223(1-2), 29-47.

Santurette, S., Poelmans, H., Luts, H., Ghesquiére, P., Wouters, J., and Dau, T. (2010). "Detection and identification of monaural and binaural pitch contours in dyslexic listeners," J. Assoc. Res. Otoloaryngol. 125(11), $515-524$.

Saunders, G. H., and Haggard, M. P. (1989). "The clinical assessment of obscure auditory dysfunction-1. Auditory and psychological factors," Ear Hear. 10, 200-208.

Schweder, T., and Spjøtvoll, E. (1982). "Plots of $P$-values to evaluate many tests simultaneously," Biometrika 69(3), 493-502.

Staffel, J. G., Hall, J. W., Grose, J. H., and Pillsbury, H. C. (1990). "NoSo and $\operatorname{NoS} \pi$ detection as a function of masker bandwidth in normal-hearing and cochlear-impaired listeners," J. Acoust. Soc. Am. 87(4), 1720-1727.

Strelcyk, O., and Dau, T. (2009). "Relations between frequency selectivity, temporal finestructure processing, and speech reception in impaired hearing," J. Acoust. Soc. Am. 125(5), 3328-3345.

Tyler, R. S., Wood, E. J., and Fernandes, M. (1983). "Frequency resolution and discrimination of constant and dynamic tones in normal and hearingimpaired listeners," J. Acoust. Soc. Am. 74(4), 1190-1199.

van Rooij, J. C. G. M., Plomp, R., and Orlebeke, J. F. (1989). "Auditive and cognitive factors in speech perception by elderly listeners. I: Development of test battery," J. Acoust. Soc. Am. 86(4), 1294-1309.

van Rooij, J. C. G. M., and Plomp, R. (1990). "Auditive and cognitive factors in speech perception by elderly listeners. II: Multivariate analyses," J. Acoust. Soc. Am. 88(6), 2611-2624.

Wagener, K., Josvassen, J. L., and Ardenkjær, R. (2003). "Design, optimization and evaluation of a Danish sentence test in noise," Int. J. Audiol. 42, $10-17$. 